\title{
FRONT PROPAGATION AT THE NEMATIC-ISOTROPIC TRANSITION TEMPERATURE*
}

\author{
APALA MAJUMDAR ${ }^{\dagger}$, PAUL A. MILEWSKI ${ }^{\dagger}$, AND AMY SPICER ${ }^{\dagger}$
}

\begin{abstract}
We study the gradient flow model for the Landau-de Gennes energy functional for nematic liquid crystals at the nematic-isotropic transition temperature on prototype geometries. We study the dynamic model on a three-dimensional droplet and on a disc with Dirichlet boundary conditions and different types of initial conditions. In the case of a droplet with radial boundary conditions, a large class of physically relevant initial conditions generate dynamic solutions with a well-defined nematic-isotropic interface which propagates according to mean curvature for small times. On a disc, we make a distinction between "planar" and "nonplanar" initial conditions, and "minimal" and "nonminimal" Dirichlet boundary conditions. Planar initial conditions generate solutions with an isotropic core for all times, whereas nonplanar initial conditions generate solutions which escape into the third dimension. Nonminimal boundary conditions generate solutions with boundary layers, and these solutions can either have a largely ordered interior profile or an almost entirely disordered isotropic interior profile. Our examples suggest that while critical points of the Landau-de Gennes energy typically have highly localized disordered-ordered interfaces, the transient dynamics exhibits observable interfaces of potential experimental relevance.
\end{abstract} faces

Key words. Landau-de Gennes theory, nematic-isotropic temperature, nematic-isotropic inter-

AMS subject classifications. 49-XX, 35-XX, 35BXX

DOI. $10.1137 / 15 \mathrm{M} 1039250$

1. Introduction. Nematic liquid crystals are classical examples of mesophases between conventional solid and liquid phases; they are anisotropic liquids with preferred directions of molecular alignment, these directions being referred to as "directors" in the literature $[1,2]$. In other words, nematic liquid crystals are complex liquids with orientational order. Nematics in confinement are an exciting source of challenges for mathematicians and practical scientists alike. We study dynamically metastable nematic configurations with interfaces in prototype geometries within the Landau-de Gennes (LdG) theory for nematic liquid crystals. In this framework, the nematic state is described by the $\mathbf{Q}=\left\{Q_{i j}\right\}$-tensor, a symmetric, traceless $3 \times 3$ matrix, interpreted as a macroscopic measure of the nematic order [1, 2]. The LdG energy functional comprises a bulk potential, determining nematic order as a function of temperature, and an elastic energy density which penalizes spatial inhomogeneities. We work at the nematic-isotropic transition temperature, where both the isotropic and nematic phases are minimizers of the bulk potential, and with the one-constant elastic energy density in the limit of the vanishing elastic constant. This limit, describing macroscopic domains (see [3]) with length scales much larger than the nematic correlation length, is studied in detail in the context of energy minimizers in [4].

We adopt the gradient flow model to describe the nematodynamics in the ab-

${ }^{*}$ Received by the editors September 11, 2015; accepted for publication (in revised form) March 22, 2016; published electronically July 19, 2016.

http://www.siam.org/journals/siap/76-4/M103925.html

${ }^{\dagger}$ Department of Mathematical Sciences, University of Bath, Bath, BA2 7AY, United Kingdom (a.majumdar@bath.ac.uk, P.A.Milewski@bath.ac.uk, A.Spicer@bath.ac.uk). The first author's work was supported by EPSRC Career Acceleration Fellowships EP/J001686/1 and EP/J001686/2 and an OCIAM Visiting Fellowship. The second author's work was supported by a Royal Society Wolfson award. The third author's work was supported by an Engineering and Physical Sciences Research Council (EPSRC) studentship. 
sence of fluid flow at a constant temperature. Gradient flows are evolution equations driven by a decreasing energy [5]. Our model is derived from the LdG energy with the $L^{2}$-norm as the dissipation mechanism and is described by a system of five coupled nonlinear parabolic partial differential equations. Standard theory for parabolic systems shows that we have a unique "dynamic" time-dependent solution for physically relevant initial and boundary conditions. Gradient flow models have been previously used in the context of liquid crystal (LC) dynamics. For example, in $[6,7]$ the authors study a one-dimensional gradient flow model and the effects of biaxiality and elastic anisotropy. In [8], the authors study nematic-isotropic front propagation using the method of matched asymptotic expansions within the more general Beris-Edwards theory for nematodynamics [9]. In particular, they account for fluid flow and the coupling between fluid flow and nematic order. They derive evolution laws for the velocity field, the director field of nematic alignment, and the nematic-isotropic interface, but without any special attention to the effects of boundary conditions and initial conditions. We work in a simpler dynamical framework with no fluid flow but with focus on how the dynamics is affected by the choice of boundary and initial data.

At the nematic-isotropic transition temperature, the LdG bulk potential bears strong resemblance to the Ginzburg-Landau (GL) potential in superconductivity. In our first model problem we study a three-dimensional droplet with Dirichlet radial boundary conditions. We use the concept of "normalized energy" for the GL gradient flow model in $[10,11]$ to prove that nematic-isotropic interfaces propagate according to mean curvature in certain model situations. The long-time dynamics is described by an explicit critical point of the LdG energy - the radial hedgehog $(\mathrm{RH})$ solution $[12,13,14]$. The $\mathrm{RH}$ solution has perfect radial symmetry, with perfect radial nematic alignment and an isolated isotropic point at the center, referred to in the literature as a point defect [13]. We focus on the interplay between initial conditions and transient dynamics followed by convergence to the static RH solution using four different representative initial conditions. The transient dynamics has some universal features which may have experimental repurcussions.

In section 4, our second model problem focuses on dynamic solutions on a disc with Dirichlet conditions, subject to two distinct types of initial conditions: planar and nonplanar. Planar $\mathbf{Q}=\left\{Q_{i j}\right\}$-tensors, $i, j=1,2,3$, have zero $Q_{13}$ and $Q_{23}$ components and nonplanar $\mathbf{Q}$-tensors do not. Using standard techniques, we prove that planar initial conditions evolve to planar dynamic solutions which have an isotropic point at the center of the disc for all times. These solutions develop a nematic-isotropic interface which propagates inwards and is arrested near the origin. Nonplanar initial conditions, including small "nonplanar" perturbations of planar initial conditions, converge to a universal nonplanar profile. The "small-time" dynamics is almost indistinguishable from the planar case; however, the interface collapses at the origin, and the dynamic solution escapes into an entirely ordered nonplanar state. We track the transient dynamics and numerically compute quantitative estimates for the "persistence time" of the interface.

These numerical results are complemented by some analysis for radially symmetric planar critical points of the LdG energy that have been reported in [15] for low temperatures. We generalize some of the results in [15] to the nematic-isotropic transition temperature and use these critical points to construct radially symmetric and nonsymmetric initial conditions for the numerical simulations. The different types of initial conditions suggest that the transient dynamics has universal features independent of the symmetry or uniaxiality/biaxiality of the initial condition. Namely, in all cases, we have a well-defined nematic-isotropic interface as a pronounced feature 
of the evolution trajectory, which we illustrate by the eigenvalue evolution of the LdG Q-tensor and plots of $|\mathbf{Q}|^{2}$ as a function of time.

The first two model problems have minimal boundary conditions which are minimizers of the LdG bulk potential. In the last section, we study two-dimensional (2D) and three-dimensional (3D) LdG dynamic solutions on a disc with nonminimal boundary conditions. The 2D case can be easily understood, and all dynamic solutions exhibit a rapidly growing isotropic core with a thin boundary layer near the lateral surface. The 3D solution landscape is richer, and the transient dynamics is sensitive to the initial condition. If the initial condition is planar with an nematic-isotropic interface relatively close to the center of the disc, then the interface propagates towards the center, replicating the planar dynamics in section 4 . If the planar initial condition has an nematic-isotropic interface relatively close to the boundary of the disc, the interface propagates outwards, yielding an almost entirely isotropic interior and replicating the 2D dynamics. In all cases, we have a boundary layer to match the fixed nonminimal Dirichlet condition. Here, the transient dynamics is sensitive to the initial interface location, a feature which is missing in the model problems with minimal Dirichlet boundary conditions.

The paper is organized as follows. In section 2, we present the gradient flow model for the Landau-de Gennes energy. In section 3, we study dynamic solutions on a droplet with Dirichlet radial conditions. Section 4 follows, with emphasis on planar and nonplanar initial conditions on a disc, and section 5 illustrates the diverse possibilities with nonminimal boundary conditions. We conclude in section 6 with future perspectives.

2. Preliminaries. The LdG $\mathbf{Q}$-tensor order parameter is in the space of symmetric traceless $3 \times 3$ matrices, $S_{0}=\left\{\mathbf{Q} \in \mathbb{M}^{3 \times 3}: Q_{i j}=Q_{j i}, Q_{i i}=0\right\}$. A $\mathbf{Q}$-tensor is said to be (i) isotropic if $\mathbf{Q}=0$, (ii) uniaxial if $\mathbf{Q}$ has a pair of degenerate nonzero eigenvalues, and (iii) biaxial if $\mathbf{Q}$ has three distinct eigenvalues $[1,16]$. A uniaxial Q-tensor can be written as $\mathbf{Q}_{u}=s(\mathbf{n} \otimes \mathbf{n}-\mathrm{I} / 3)$ with $s \in \mathbb{R}$ and $\mathbf{n} \in S^{2}$ a unit vector. The scalar, $s$, is an order parameter which measures the degree of orientational order. The vector, $\mathbf{n}$, is referred to as the "director" and labels the single distinguished direction of uniaxial nematic alignment $[2,1]$.

We work with a simple form of the LdG energy given by

$$
I[\mathbf{Q}]=\int_{\Omega} \frac{L}{2}|\nabla \mathbf{Q}|^{2}+f_{B}(\mathbf{Q}) \mathrm{d} V,
$$

where

$$
|\nabla \mathbf{Q}|^{2}=\frac{\partial \mathbf{Q}_{i j}}{\partial \mathbf{x}_{k}} \frac{\partial \mathbf{Q}_{i j}}{\partial \mathbf{x}_{k}}, \quad f_{B}(\mathbf{Q})=\frac{A}{2} \operatorname{tr} \mathbf{Q}^{2}-\frac{B}{3} \operatorname{tr} \mathbf{Q}^{3}+\frac{C}{4}\left(\operatorname{tr} \mathbf{Q}^{2}\right)^{2}
$$

The variable $A=\alpha\left(T-T^{*}\right)$ is the rescaled temperature, $\alpha, L, B, C>0$ are material-dependent constants, and $T^{*}$ is the characteristic nematic supercooling temperature $[1,16]$. Further, $\operatorname{tr} \mathbf{Q}^{2}=Q_{i j} Q_{i j}$ and $\operatorname{tr} \mathbf{Q}^{3}=Q_{i j} Q_{j k} Q_{k i}$ for $i, j, k=1,2,3$. It is well known that all stationary points of the thermotropic potential, $f_{B}$, are either uniaxial or isotropic $[1,16,17]$. The rescaled temperature $A$ has three characteristic values: (i) $A=0$, below which the isotropic phase $\mathbf{Q}=0$ loses stability; (ii) the nematic-isotropic transition temperature, $A=B^{2} / 27 C$, at which $f_{B}$ is minimized by the isotropic phase and a continuum of uniaxial states with $s=s_{+}=B / 3 C$ and $\mathbf{n}$ arbitrary; and (iii) the nematic supercooling temperature, $A=B^{2} / 24 C$, above which the ordered nematic equilibria do not exist. 
Throughout the paper we work at the nematic-isotropic transition temperature, investigating the propagation of fronts separating the isotropic phase from the ordered nematic phase in the limit, $L C / R^{2} B^{2} \rightarrow 0^{+}$, where $R$ is a characteristic length scale of the domain $\Omega$. We refer to this as the vanishing elastic constant limit for fixed values of $R, B, C$, by analogy with the terminology in [4]. Continuum formulations are typically valid in this limit $[12,3]$. We work with the gradient flow model associated with the LdG energy [18], and the dynamic equations are given by

$$
\gamma \mathbf{Q}_{t}=L \Delta \mathbf{Q}-A \mathbf{Q}+B\left(\mathbf{Q Q}-\frac{1}{3}|\mathbf{Q}|^{2} \mathbf{I}\right)+C|\mathbf{Q}|^{2}|\mathbf{Q}|,
$$

where $\gamma$ is a positive rotational viscosity, $\mathbf{Q Q}=\mathbf{Q}_{i j} \mathbf{Q}_{j k}$ with $i, j, k=1,2,3$, and $\mathbf{I}$ is the $3 \times 3$ identity matrix. The system (2.3) comprises five coupled nonlinear parabolic partial differential equations. We recall the following basic result about the existence and uniqueness of solutions for such gradient flow systems.

Proposition 2.1. Let $\Omega \subset \mathbb{R}^{3}$ be a bounded domain with smooth boundary, $\partial \Omega$. Given a smooth fixed boundary condition $\mathbf{Q}(\mathbf{x}, t)=\mathbf{Q}_{b}(r)$ on $\partial \Omega$ and smooth initial condition $\mathbf{Q}(\mathbf{x}, 0)=\mathbf{Q}_{0}(\mathbf{x})$, the parabolic system (2.3) has a unique solution, $\mathbf{Q}(\mathbf{x}, t) \in$ $C^{\infty}(\Omega)$ for all $t>0$.

Proof. The existence of a solution is standard; see [19] for a proof. From [20, 4], we have that the dynamic solution is bounded for all times with $|\mathbf{Q}(\mathbf{r}, t)| \leq \sqrt{2 / 3} B / 3 C$ for $t \geq 0$. The uniqueness result follows from an immediate application of Gronwall's inequality to the difference $\mathbf{Q}_{d}=\mathbf{Q}_{1}-\mathbf{Q}_{2}$ of two solutions, $\mathbf{Q}_{1}$ and $\mathbf{Q}_{2}$, subject to the fixed boundary condition and the same initial condition. In particular, $\mathbf{Q}_{d}(\mathbf{x}, t)=0$ on $\partial \Omega$ and $\mathbf{Q}_{d}(\mathbf{x}, 0)=0$ for $\mathbf{x} \in \Omega$. One can then show that $\mathbf{Q}_{d}(\mathbf{x}, t)=0$ for $\mathbf{x} \in \Omega$ and for all $t>0$.

3. Front propagation on $3 \mathrm{D}$ spherical droplets. Our first example concerns nematic droplets. Let $\Omega$ be the unit ball in three dimensions; $\Omega:=\left\{\mathbf{x} \in \mathbb{R}^{3} ;|\mathbf{x}| \leq 1\right\}$. We work with a uniaxial Dirichlet boundary condition,

$$
\mathbf{Q}_{b}=\frac{B}{3 C}\left(\hat{\mathbf{r}} \otimes \hat{\mathbf{r}}-\frac{\mathbf{I}}{3}\right),
$$

where $\hat{\mathbf{r}}$ is the $3 \mathrm{D}$ radial unit vector. $\mathbf{Q}_{b}$ is a minimizer of the bulk potential $f_{B}$ in (2.2). For illustration, we first work with uniaxial radial initial conditions that have a front structure such as

$$
\mathbf{Q}(\mathbf{x}, 0)= \begin{cases}\mathbf{0}, & 0<|\mathbf{x}|<r_{0} \\ \mathbf{Q}_{b}, & r_{0}<|\mathbf{x}| \leq 1,\end{cases}
$$

for some $\frac{1}{2} \leq r_{0}<1$. We refer to these as "radial hedgehog"-type initial conditions by analogy with the static RH solution, as described in section 1. We are interested in the qualitative properties of dynamic solutions of (2.3) subject to these initial and boundary conditions, such as front propagation and transient dynamics. Looking for "dynamic" RH-type solutions, we work with an ansatz of the form

$$
\mathbf{Q}(\mathbf{r}, t)=h(r, t)\left(\hat{\mathbf{r}} \otimes \hat{\mathbf{r}}-\frac{\mathbf{I}}{3}\right),
$$

where $h:[0,1] \times[0, \infty) \rightarrow \mathbb{R}$ is the scalar order parameter that depends only on $r$, the radial distance from the origin, and time. On substitution into (2.3), we have a 
solution of the form (3.3) if the scalar order parameter $h$ is a solution of

$$
\gamma h_{t}=h_{r r}+\frac{2}{r} h_{r}-\frac{6 h}{r^{2}}+\frac{3}{\bar{L}} h\left(h_{+}-h\right)\left(2 h-h_{+}\right),
$$

with $h_{+}=\frac{B}{3 C}$ and $\bar{L}=\frac{9 L}{C}$, where $\gamma$ is a rescaled viscosity coefficient. The boundary conditions are $h(0, t)=0, h(1, t)=h_{+}$for all $t \geq 0$, and the initial condition is

$$
h(r, 0)= \begin{cases}0, & 0 \leq r<r_{0} \\ h_{+}, & r_{0}<r<1\end{cases}
$$

The evolution equation for $h$ in (3.4) is simply the gradient flow model associated with the energy functional

$$
\frac{I[h]}{4 \pi \sqrt{\bar{L}}}=\int_{0}^{1} r^{2} \sqrt{\bar{L}}\left\{\frac{1}{3}\left(\frac{d h}{d r}\right)^{2}+\frac{2 h^{2}}{r^{2}}+\frac{h^{2}\left(h-h_{+}^{2}\right)^{2}}{\sqrt{L}}\right\} \mathrm{d} r .
$$

Given a smooth solution $h(r, t) \in C^{\infty}([0,1] \times[0, \infty))$, we can appeal to the uniqueness result in Proposition 2.1 to deduce that (3.3) is the physically relevant solution, and hence the $5 \mathrm{D}$ evolution problem in (2.3) reduces to a single evolution equation for a scalar order parameter.

In [10], the authors study a closely related problem for front propagation on 3D balls in the GL framework. They rigorously prove that for suitably defined initial conditions (as in (3.5)) with appropriately bounded energy, the front propagates according to mean curvature. Our governing equation (3.4) is similar to that studied in section 3 of [10]; however, we have an extra term: $-6 h / r^{2}$ in (3.4). In particular, we cannot quote results from [10] and [11] without verifying that the key inequalities are unchanged by the additional term for $\bar{L}$ sufficiently small. In the next few paragraphs, we verify the necessary details to reach the desired conclusion. Let $\rho(t)$ be the solution to

$$
\frac{d \rho}{d t}=-\frac{2}{\rho}, \quad \rho(0)=r_{0} \in\left(\frac{1}{2}, 1\right)
$$

or alternatively, $\rho(t)=\sqrt{r_{0}^{2}-4 t}$. We define $T_{1}=\frac{1}{4}\left(r_{0}^{2}-\frac{1}{4}\right)$. This is the first time for which $\rho(t)=\frac{1}{2}$ and is independent of $\bar{L}$. Next, let

$$
f(r, t)= \begin{cases}0, & 0 \leq r \leq \rho(t) \\ h_{+}, & \rho(t) \leq r \leq 1\end{cases}
$$

Our goal is to show that the solution $h(r, t)$ of $(3.4)$, subject to suitably defined initial conditions, resembles the function $f(r, t)$ for $T<T_{1}$, in the sense that

$$
\int_{0}^{1} r^{2}|h(r, t)-f(r, t)| \mathrm{d} r \rightarrow 0 \quad \text { as } \bar{L} \rightarrow 0 .
$$

As in [10], the key step is to define a weighted energy as shown below:

$$
E_{\phi}[w](\tau)=\int_{-\rho(\tau)}^{1-\rho(\tau)} \phi(R, \tau)\left\{\sqrt{\bar{L}}\left(\frac{w_{R}^{2}}{3}+\frac{2 w^{2}}{(R+\rho)^{2}}\right)+\frac{w^{2}\left(h_{+}-w\right)^{2}}{\sqrt{\bar{L}}}\right\} \mathrm{d} R
$$


where

$$
w(R, \tau)=h(R+\rho(t), t), \quad-\rho(\tau) \leq R \leq 1-\rho(\tau), \quad \tau \geq 0,
$$

and $\phi(R, \tau)$ is a weight function

$$
\phi(R, \tau)=\exp \left[-\frac{2 R}{\rho}\right]\left(1+\frac{R}{\rho}\right)^{2} .
$$

In particular, $w(R, \tau)$ is a solution of

$$
\sqrt{\bar{L}} w_{\tau}-\frac{\sqrt{\bar{L}}}{\phi}\left(\phi w_{R}\right)_{R}+\frac{6 w \sqrt{\bar{L}}}{(R+\rho)^{2}}+\frac{3}{\sqrt{\bar{L}}} w\left(h_{+}-w\right)\left(2 w-h_{+}\right)=0 .
$$

We follow the steps in Proposition 3.2 of [10] to show that

$$
\frac{d}{d \tau} E_{\phi}[w](\tau) \leq-\frac{2}{3} \frac{\sqrt{L}}{\gamma} \int_{-\rho(\tau)}^{1-\rho(\tau)} \phi(R, \tau) w_{\tau}^{2} \mathrm{~d} R+\frac{4 \phi}{\rho} \bar{L}^{1 / 2} h_{+}^{2}
$$

for $\tau \leq T_{1}$. By contrast, in [10], the weighted energy in the GL framework is strictly decreasing. We have lesser control on the weighted energy but the inequality (3.13) suffices for our purposes, for $\bar{L}$ sufficiently small.

Next, we define an interface energy which yields lower bounds for the weighted energy

$$
g(s)=\frac{2}{\sqrt{3}} \int_{0}^{s} w\left(h_{+}-w\right) \mathrm{d} w
$$

and so $g\left(h_{+}\right)=\frac{h_{+}^{3}}{3 \sqrt{3}}$ is an interface energy associated with an nematic-isotropic front. Further, let

$$
v(R)= \begin{cases}0, & -\rho(\tau) \leq R<0, \\ h_{+}, & 0<R<1-\rho(\tau) .\end{cases}
$$

We can adapt a lemma in [11] to show the following result.

Proposition 3.1. If for some smooth function $w$,

$$
\int_{-a}^{a}|g(w)-g(v)| \mathrm{d} s \leq \frac{g\left(h_{+}\right)}{4} \bar{L}^{\alpha} \quad \text { and } \quad E_{\phi}[w] \leq C_{1},
$$

where $0<\alpha<1 / 4$ and $a=\rho\left(T_{1}\right) / 2 \sqrt{2}$, then $E_{\phi}[w](\tau) \geq g\left(h_{+}\right)-C_{2} \bar{L}^{1 / 2-\alpha}-C_{3} \bar{L}^{2 \alpha}$ for $\tau \leq T_{1}$ and positive constants $C_{1}, C_{2}, C_{3}$ independent of $\bar{L}$.

It remains to construct initial conditions $w(R, 0)$, which satisfy the hypothesis of Proposition 3.1. The construction is parallel to that in [21, eq. (1.22)], and we give a statement for completeness.

Proposition 3.2. Define the function

$$
\sigma(R)=\frac{h_{+}}{1+\exp \left[-\sqrt{3} h_{+} R\right]} .
$$


At $\tau=0, R=r-r_{0}$, and for $\bar{L}$ sufficiently small, define

$$
w(R)= \begin{cases}h_{+}, & R>2 \bar{L}^{1 / 4}, \\ \left(h_{+}-\sigma\left(\frac{1}{L^{1 / 4}}\right)\right) \frac{R-2 \bar{L}^{1 / 4}}{L^{1 / 4}}+h_{+}, & \bar{L}^{1 / 4} \leq R \leq 2 \bar{L}^{1 / 4}, \\ \sigma\left(\frac{R}{\sqrt{L}}\right), & -\bar{L}^{1 / 4} \leq R \leq \bar{L}^{1 / 4}, \\ \sigma\left(-\frac{1}{L^{1 / 4}}\right) \frac{R+2 \bar{L}^{1 / 4}}{L^{1 / 4}}, & -2 \bar{L}^{1 / 4} \leq R \leq-\bar{L}^{1 / 4}, \\ 0, & R<-2 \bar{L}^{1 / 4} .\end{cases}
$$

Then $E_{\phi}[w] \leq g\left(h_{+}\right)+C \bar{L}^{1 / 4}$ for a positive constant $C$ independent of $\bar{L}$ as $\bar{L} \rightarrow 0$.

Finally, we have the next proposition, by analogy with the main theorem in [11].

Proposition 3.3. Let $0<\alpha \leq \frac{1}{4}$, and assume that $E_{\phi}[w](0) \leq g\left(h_{+}\right)+c_{1} \bar{L}^{2 \alpha}$ for some constant $c_{1}>0$ independent of $\bar{L}$ and that, for a as above,

$$
\int_{-a}^{a}|g(w(R, 0))-g(v)| \mathrm{d} R<\frac{g\left(h_{+}\right)}{8} \bar{L}^{\alpha} .
$$

Let $T_{\epsilon}$ be the first time for which

$$
\int_{-a}^{a}\left|g\left(w\left(R, T_{\epsilon}\right)\right)-g(w(R, 0))\right| \mathrm{d} R=\frac{g\left(h_{+}\right)}{8} \bar{L}^{\alpha} .
$$

Then $T_{\epsilon} \geq \min \left(T_{1}, C\right)$ for some positive constant $C$ independent of $\bar{L}$ as $\bar{L} \rightarrow 0^{+}$. In other words, we have

$$
\int_{-a}^{a}|g(w(R, \tau))-g(v)| \mathrm{d} R<\frac{g\left(h_{+}\right)}{4} \bar{L}^{\alpha}
$$

for all $\tau<T_{\epsilon}$, and $T_{\epsilon}$ is of order one.

The proof follows verbatim from [11]. Equipped with a weighted energy, estimates for the rate of change of the weighted energy, and bounds for the weighted energy along with suitable initial conditions, we adapt arguments from Theorem 3.1 of [10] to prove the next result.

Theorem 3.4. Let $\Omega$ be the unit ball in $\mathbb{R}^{3}$. Let $h_{\bar{L}}(r, t)$ denote the solution of the evolution equation (3.4), subject to the fixed boundary conditions and an initial condition with an interface structure and appropriately bounded weighted energy:

$$
\int_{0}^{1} \psi_{0}(r)\left[\sqrt{\bar{L}}\left(\frac{w_{R}^{2}}{3}+\frac{2 w^{2}}{(R+\rho)^{2}}\right)+\frac{w^{2}\left(h_{+}-w\right)^{2}}{\sqrt{\bar{L}}}\right] r^{2} \mathrm{~d} r \leq g\left(h_{+}\right)+\Gamma \bar{L}^{1 / 4}
$$

with $\Gamma$ independent of $\bar{L}$ and $\psi_{0}(r)=\frac{1}{r_{0}^{2}} \exp \left[-2\left(\frac{r}{r_{0}}-1\right)\right]$. Then for any $T<T_{\epsilon}$, where $T_{\epsilon}$ has been defined in Proposition 3.3, we have

$$
\lim _{\bar{L} \rightarrow 0} \int_{0}^{T} \int_{\Omega}\left|h_{\bar{L}}(r, t)-f(r, t)\right| r^{2} \mathrm{~d} r \mathrm{~d} t=0,
$$

where $f(r, t)$ has been defined in (3.8). 
Comments on the proof. The condition (3.22) is equivalent to the bound in Proposition 3.2, which can be realized by initial conditions with "efficient interfaces." The key ingredients are the rate of change of the weighted energy in (3.13), the lower bound for the weighted energy in terms of $g\left(h_{+}\right)$in Proposition 3.1, and the upper bound in (3.22). These ensure that the system does not have sufficient energy to create additional interfaces away from $R=\rho(\tau)$ and that $h$ is effectively constant (either $h=0$ or $h=h_{+}$) away from $R=\rho(\tau)$. Given $h_{\bar{L}}(r, t)$, we obtain the unique solution $\mathbf{Q}_{\bar{L}}(\mathbf{x}, t)$ of the evolution equation (2.3), subject to the boundary and initial conditions, given by $\mathbf{Q}_{\bar{L}}(\mathbf{x}, t)=h_{\bar{L}}(|\mathbf{x}|, t)\left(\hat{\mathbf{r}} \otimes \hat{\mathbf{r}}-\frac{\mathbf{I}}{3}\right)$.

3.1. Numerical simulations on the sphere. We numerically compute solutions of the gradient flow system (2.3) on a 3D droplet, with the fixed boundary condition $\mathbf{Q}_{b}$ in (3.1) and various types of initial conditions. From the numerical results in [13], the radial hedgehog $(\mathrm{RH})$ solution is the global minimizer of the LdG energy for this model problem; the RH solution is a uniaxial solution of the form $\mathbf{H}=s(r)\left(\hat{\mathbf{r}} \otimes \hat{\mathbf{r}}-\frac{\mathbf{I}}{3}\right)$, where $s(0)=0$ and $s(r)>0$ for $r>0$. In particular, $s$ rapidly interpolates between $s=0$ and the boundary value of $s_{+}=B / 3 C$ over a distance proportional to the nematic correlation length, and the localized region of reduced order near $r=0$ is referred to as the "defect core." We expect the long-time dynamics to converge to the RH solution for all choices of initial conditions. However, we are equally interested in the transient dynamics and the dynamic persistence of nematic-isotropic interfaces. In what follows, we look at four different initial conditions: uniaxial initial conditions within the remit of Theorem 3.4, uniaxial initial conditions outside the scope of Theorem 3.4, biaxial initial conditions, and initial conditions that break the radial symmetry of the order parameter.

Let $R$ be the radius of the droplet; we nondimensionalize system (2.3) by setting $\bar{t}=\frac{20 t L}{\gamma R^{2}}, \overline{\mathbf{x}}=\frac{\mathbf{x}}{R}$ to yield

$$
\frac{\partial Q_{11}}{\partial \bar{t}}=\bar{\Delta} Q_{11}-\frac{1}{\tilde{L}}\left(A Q_{11}+2 C\left(Q_{11}^{2}+Q_{22}^{2}+Q_{12}^{2}+Q_{11} Q_{22}+Q_{13}^{2}+Q_{23}^{2}\right) Q_{11}\right.
$$

$$
\left.-\frac{B}{3}\left(Q_{11}^{2}+Q_{12}^{2}+Q_{13}^{2}-2 Q_{22}^{2}-2 Q_{11} Q_{22}-2 Q_{23}^{2}\right)\right)
$$

$$
\frac{\partial Q_{22}}{\partial \bar{t}}=\bar{\Delta} Q_{22}-\frac{1}{\tilde{L}}\left(A Q_{22}+2 C\left(Q_{11}^{2}+Q_{22}^{2}+Q_{12}^{2}+Q_{11} Q_{22}+Q_{13}^{2}+Q_{23}^{2}\right) Q_{22}\right.
$$

$$
\frac{\partial Q_{12}}{\partial \bar{t}}=\bar{\Delta} Q_{12}-\frac{1}{\tilde{L}}\left(A Q_{12}+2 C\left(Q_{11}^{2}+Q_{22}^{2}+Q_{12}^{2}+Q_{11} Q_{22}+Q_{13}^{2}+Q_{23}^{2}\right) Q_{12}\right.
$$

$$
\left.-B\left(Q_{11} Q_{12}+Q_{12} Q_{22}+Q_{13} Q_{23}\right)\right)
$$

$$
\frac{\partial Q_{13}}{\partial \bar{t}}=\bar{\Delta} Q_{13}-\frac{1}{\tilde{L}}\left(A Q_{13}+2 C\left(Q_{11}^{2}+Q_{22}^{2}+Q_{12}^{2}+Q_{11} Q_{22}+Q_{13}^{2}+Q_{23}^{2}\right) Q_{13}\right.
$$

$$
\left.-B\left(Q_{12} Q_{23}-Q_{22} Q_{13}\right)\right)
$$

$$
\frac{\partial Q_{23}}{\partial \bar{t}}=\bar{\Delta} Q_{23}-\frac{1}{\tilde{L}}\left(A Q_{23}+2 C\left(Q_{11}^{2}+Q_{22}^{2}+Q_{12}^{2}+Q_{11} Q_{22}+Q_{13}^{2}+Q_{23}^{2}\right) Q_{23}\right.
$$

$$
\left.-B\left(Q_{12} Q_{13}-Q_{23} Q_{11}\right)\right)
$$



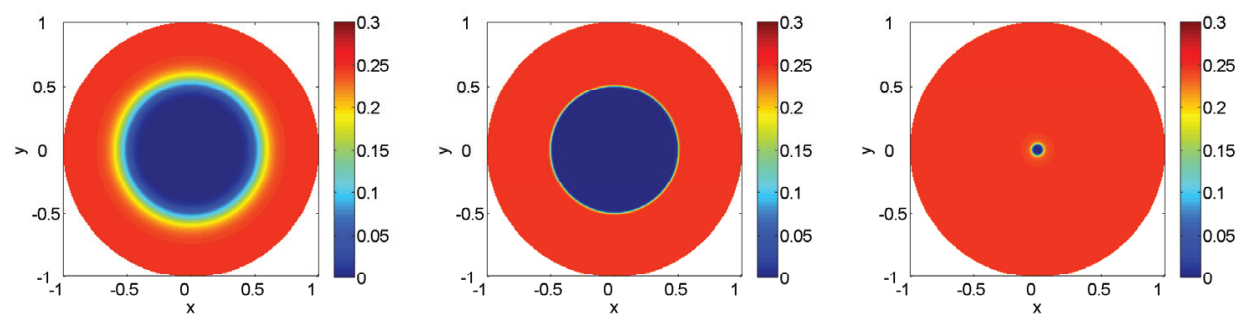

FIG. 3.1. $|\boldsymbol{Q}(\mathbf{r}, t)|^{2}$ on the cross section of the unit ball at $\phi=0$ for Case I and Case II, at $t=0, t=0.001$, and $t=0.125$. The spatial resolution is $h=\frac{1}{256}$.

where $\bar{\Delta}$ denotes the Laplacian with respect to the rescaled coordinate $\overline{\mathbf{x}}$. (In what follows, we drop the bars from the dimensionless variables.) We take $R^{2}=10^{-10} \mathrm{~m}^{2}$, $\tilde{L}=\frac{L}{R^{2}} \mathrm{~N} / \mathrm{m}^{2}, B=0.64 \times 10^{4} \mathrm{~N} / \mathrm{m}^{2}, C=0.35 \times 10^{4} \mathrm{~N} / \mathrm{m}^{2}$, and $A=\frac{B^{2}}{27 C}$ throughout the paper and work with either $\tilde{L}=0.05$ or 0.01 [13].

Here, and in the subsequent sections of this paper, the system of reaction-diffusion equations (3.24)-(3.28) is solved as follows. The unit ball is embedded into the unit cube $[-1,1]^{3}$, which is discretized with a uniform Cartesian grid with spatial resolution $h$. We implement a special case of an immersed boundary method (see, for example, [22]) and apply the boundary conditions at all discrete points within distance $\frac{h}{2}$ of the boundary. For interior points, the solution satisfies the system (3.24)-(3.28), and in the exterior of the physical domain we solve the simple heat equation (i.e., take $A=B=C=0$ ) and use periodic boundary conditions on the cube. This setup makes it simple and efficient to use higher-order and spectral schemes for spatial derivatives. Timestepping is accomplished with a standard fourth-order Runge-Kutta scheme. Simple finite difference schemes are also implemented to verify the results.

The first two initial conditions are uniaxial RH-type initial conditions of the form

$$
\mathbf{Q}(\mathbf{r}, 0)=h(r, 0)\left(\hat{\mathbf{r}} \otimes \hat{\mathbf{r}}-\frac{\mathbf{I}}{3}\right) .
$$

Case I prescribes an initial condition, $h(r, 0)$, with an interface structure given by $h(r)=\frac{1}{2} h_{+}\left(1+\tanh \left(\left(r-r_{0}\right) / \sqrt{\tilde{L}}\right)\right)$, and Case II describes an initial condition without an interface structure given by $h(r)=h_{+} r$. Case I is within the remit of Theorem 3.4, and the numerics demonstrate that the solution retains the nematic-isotropic interface for all times and that the interface propagates towards the origin according to mean curvature for small times, equilibrating near the origin for long times. For long times, the radius of the isotropic core scales, as expected, with $\sqrt{\tilde{L}}$ and arises out of the saddle structure of $\boldsymbol{Q}$ at the origin. The dynamic solution in Case II very quickly develops an inwards-propagating interface separating the isotropic core at the center from the ordered nematic state and then follows the same evolution path as Case I (see Figures 3.1, 3.2, and 3.3). The long-time behavior of the dynamic solutions for Cases I and II are indistinguishable within numerical resolution, as expected.

For Case III, we use a biaxial initial condition given by

$$
\boldsymbol{Q}(\mathbf{r}, 0)=h(r)\left(\hat{\boldsymbol{r}} \otimes \hat{\boldsymbol{r}}-\frac{\boldsymbol{I}}{3}\right)+s(r)(\boldsymbol{m} \otimes \boldsymbol{m}-\boldsymbol{p} \otimes \boldsymbol{p}),
$$

where $\boldsymbol{m}=(\cos \theta \cos \phi, \cos \theta \sin \phi,-\sin \theta)$ and $\boldsymbol{p}=(-\sin \phi, \cos \phi, 0)$. The function $h(r)$ has an interface structure, as in Case I, and $s(r)=r(1-r)$. This case is outside 




FIG. 3.2. Interface position for Case I and Case II with $r_{0}=0.5$ (black), and predicted position according to motion by mean curvature (blue) for $\tilde{L}=0.05$ (dashed) and $\tilde{L}=0.01$ (solid). In Case II an interface quickly develops, so the two curves are indistinguishable. The spatial resolutions for $\tilde{L}=0.05$ and $\tilde{L}=0.01$ are $h=\frac{1}{128}$ and $\frac{1}{256}$, respectively.
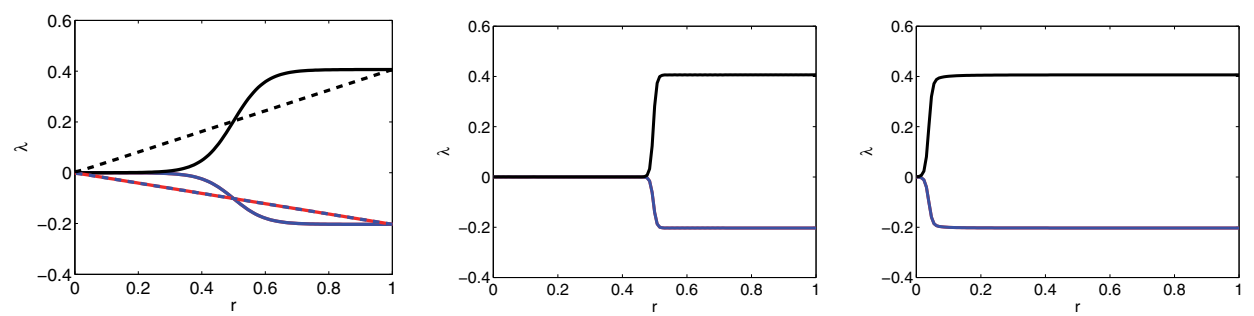

FIG. 3.3. Eigenvalues of $\boldsymbol{Q}(\mathbf{r}, t)$ as a function of $r$, for Case I and Case II (dotted), at $t=0$, $t=0.001$, and $t=0.125$.
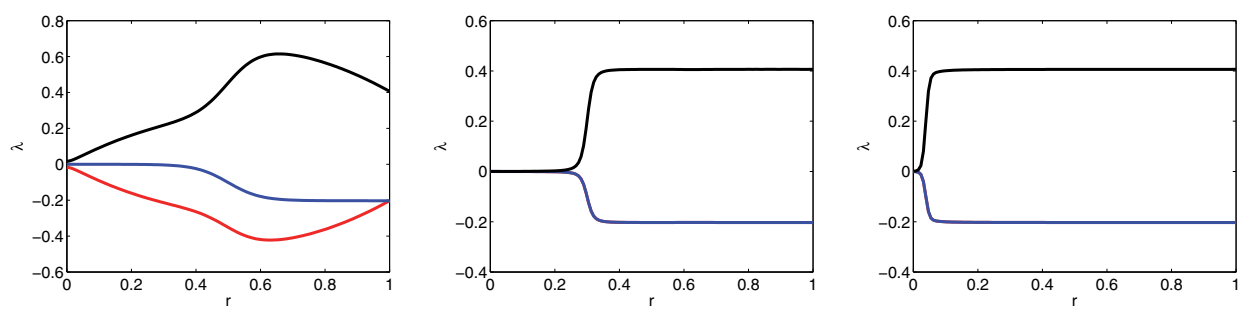

FIG. 3.4. Eigenvalues of $\boldsymbol{Q}(\mathbf{r}, t)$ as a function of $r$ for initial condition (3.30), at $t=0$, $t=1.5 \times 10^{-4}$, and $t=0.085$.

the scope of Theorem 3.4, and we are not guaranteed the uniaxial radial symmetry of the dynamic solution. The numerics show that the dynamic solution quickly becomes uniaxial within numerical resolution, as demonstrated by the evolution of the eigenvalues of $\mathbf{Q}(\mathbf{r}, t)$ in Figure 3.4. The dynamic solution exhibits an inwards-propagating interface separating the isotropic core at $r=0$ from the ordered nematic state, and the interface equilibrates near the origin. We also numerically compute the differences

$$
\left|\frac{Q_{i j}(\mathbf{r}, t)}{|\mathbf{Q}|}-\left(\frac{\mathbf{x}_{i} \mathbf{x}_{j}}{|\mathbf{r}|^{2}}-\frac{\delta_{i j}}{3}\right)\right|
$$

as a function of time, and our numerics show that this difference vanishes everywhere away from the origin, since $|\mathbf{Q}(0, t)|=0$ for large times; see Figure 3.5.

Case IV breaks the radial symmetry of the initial order parameter by employing 

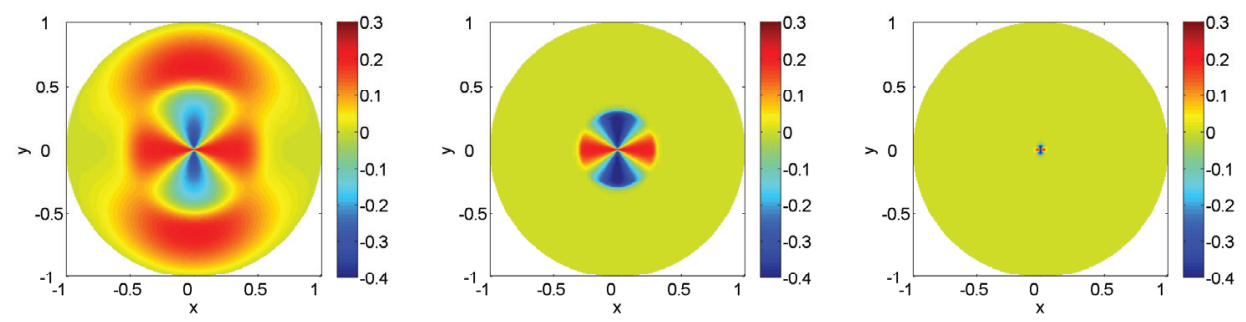

FIG. 3.5. $Q_{11}(\mathbf{r}, t)-h_{+}\left((\cos \phi \sin \theta)^{2}-\frac{1}{3}\right)$ on the cross section of the unit ball for initial condition (3.30) at $t=0$ (left), $t=1.5 \times 10^{-4}$ (middle), and $t=0.085$ (right). The spatial resolution is $h=\frac{1}{256}$.
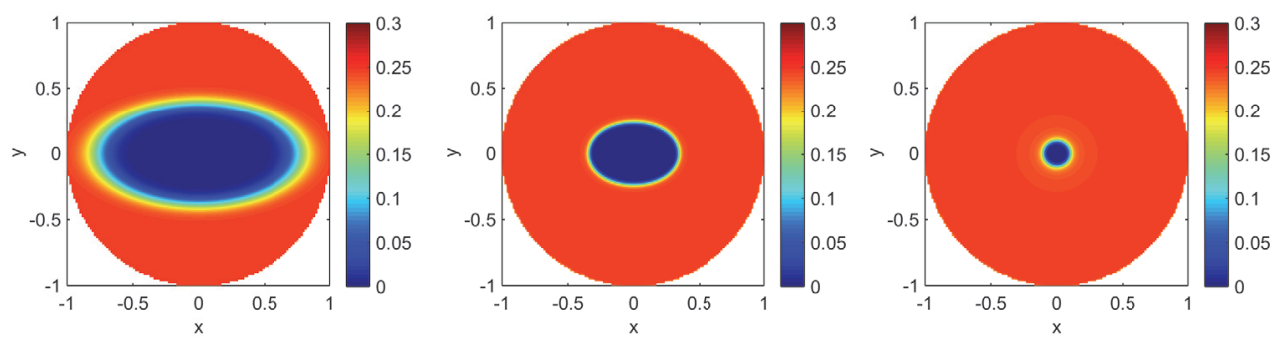

FIG. 3.6. $|\boldsymbol{Q}(\mathbf{r}, t)|^{2}$ on the cross section of the unit ball for initial condition (3.31) at $t=0$ (left), $t=0.05$ (middle), and $t=0.1$ (right). The spatial resolution is $h=\frac{1}{256}$.

a uniaxial initial condition of the form (3.29) with

$$
h(r, \theta, \phi, 0)=\frac{B}{6 C}\left(1+\tanh \left(\frac{r^{2} \sin ^{2} \theta\left(\cos ^{2} \phi+4 \sin ^{2} \phi\right)+2 r^{2} \cos ^{2} \theta-0.5}{\sqrt{\tilde{L}}}\right)\right) .
$$

Here, the initial interface is ellipsoidal in shape. The interface becomes circular and the subsequent dynamics are indistinguishable from those of Case I, as seen in Figure 3.6 .

These examples illustrate that while the static RH solution has a localized defect core of reduced order near the origin that may not be experimentally observable, the transient solutions exhibit well-defined nematic-isotropic interfaces (see Figure 3.2). These interfaces propagate towards the droplet center and may be experimentally observable.

\section{Fronts on a disc.}

4.1. Analysis on a disc. We take our computational domain to be the unit disc defined by

$$
\Omega=\left\{(r, \theta) \in \mathbb{R}^{2} ; 0 \leq r \leq 1,0 \leq \theta<2 \pi\right\},
$$

with fixed boundary condition $\mathbf{Q}=\mathbf{Q}_{c}=\frac{B}{3 C}\left(\mathbf{n}_{1} \otimes \mathbf{n}_{1}-\frac{\mathbf{I}}{3}\right)$ on $r=1$ for $\mathbf{n}_{1}=$ $(\cos \theta, \sin \theta, 0)$ - the $2 \mathrm{D}$ radial unit vector. This boundary condition is purely uniaxial and is a minimum of the bulk potential. We study dynamic solutions of the parabolic system, (2.3), subject to $\mathbf{Q}=\mathbf{Q}_{c}$ on $r=1$ with different types of initial conditions. We note that such solutions also survive as translationally invariant solutions, independent of $z$, on a cylinder with free boundary conditions on the top and bottom surfaces. 
We first present some heuristics based on critical points of the LdG energy on a disc subject to this Dirichlet condition. The gradient flow model dictates that dynamic solutions evolve along a path of decreasing energy, converging to a critical point of the LdG energy [5]. Hence, the long-time behavior can be predicted by an analysis of the corresponding stationary problem. In [4], the authors present a general analysis of LdG energy minimizers on 3D nice domains (see (2.1)) in the $L \rightarrow 0$ limit. Based on their analysis, the minimizers converge strongly in $W^{1,2}\left(\Omega ; S_{0}\right)$ to a limiting harmonic map of the form $\mathbf{Q}=s\left(\mathbf{n}^{*} \otimes \mathbf{n}^{*}-\frac{\mathbf{I}}{3}\right)$ such that $s=0$ or $s=B / 3 C$ a.e. (so that $\mathbf{Q}$ is a minimum of $f_{B}$ ) and the director $\mathbf{n}^{*}$ is a solution of the harmonic map equations $\Delta \mathbf{n}^{*}+\left|\nabla \mathbf{n}^{*}\right|^{2} \mathbf{n}^{*}=0$ (also see [23] for recent work on planar domains). The convergence is shown to be uniform away from the singularities of the limiting harmonic map, which need not be unique. There are at least two harmonic maps on a disc with the boundary condition $\mathbf{n}^{*}=(\cos \theta, \sin \theta, 0)$ on $r=1$ [24],

$$
\mathbf{n}_{1}=(\cos \theta, \sin \theta, 0) \quad \text { and } \quad \mathbf{n}_{2}=\left(\frac{2 x}{1+r^{2}}, \frac{2 y}{1+r^{2}}, \frac{1-r^{2}}{1+r^{2}}\right) .
$$

We conjecture that there are at least two competing profiles for dynamic solutions of this $2 \mathrm{D}$ problem, defined in terms of $\mathbf{n}_{1}$ and $\mathbf{n}_{2}$ above:

$$
\mathbf{Q}_{1}=s\left(\mathbf{n}_{1} \otimes \mathbf{n}_{1}-\frac{\mathbf{I}}{3}\right) \quad \text { and } \quad \mathbf{Q}_{2}=\frac{B}{3 C}\left(\mathbf{n}_{2} \otimes \mathbf{n}_{2}-\frac{\mathbf{I}}{3}\right) .
$$

As $\mathbf{n}_{1}$ is not defined at $r=0, \mathbf{Q}_{1}$ must have an isotropic point at $r=0$, with $s \rightarrow B / 3 C$ rapidly away from $r=0$. We refer to $\mathbf{Q}_{1}$ as being the $2 \mathrm{D}$ planar radial hedgehog profile. However, $\mathbf{n}_{2}$ has no singularity on $\Omega$, and $\mathbf{Q}_{2}$ does not have an isotropic core. We predict that dynamic solutions of (2.3), subject to $\mathbf{Q}=\mathbf{Q}_{c}$ on $r=1$, converge to $\mathbf{Q}_{1}$ away from the origin (where $\mathbf{n}_{1}$ is singular) if escape into the third dimension is not allowed. The dynamic solutions converge to $\mathbf{Q}_{2}$ if escape into the third dimension is allowed. Next, we have a lemma which demonstrates that escape into the third dimension is not allowed for certain initial conditions. We refer to a $\mathbf{Q}$-tensor as being "planar" if the components $Q_{13}, Q_{23}=0$ are identically zero on $\Omega$ and "nonplanar" if not. In particular, $\mathbf{Q}_{c}$, is a planar $\mathbf{Q}$-tensor.

LEMMA 4.1. Let $\mathbf{Q}(\mathbf{r}, t)$ be a solution of (2.3) on $\Omega$, with fixed boundary condition $\mathbf{Q}=\mathbf{Q}_{c}$ on $r=1$ and a planar initial condition $\mathbf{Q}(\mathbf{r}, 0)$ such that $|\mathbf{Q}(\mathbf{r}, 0)| \leq \sqrt{\frac{2}{3}}\left(\frac{B}{3 C}\right)$ for $\mathbf{r} \in \Omega$. Then $Q_{13}=Q_{23}=0$ for all $t \geq 0$.

Proof. The proof is an immediate application of Gronwall's inequality [25]. From $[20,4]$, we have the following $L^{\infty}$-bound for the dynamic solution: $|\mathbf{Q}(\mathbf{r}, t)| \leq \sqrt{\frac{2}{3}}\left(\frac{B}{3 C}\right)$ for $t \geq 0$. The two governing PDEs for $Q_{13}$ and $Q_{23}$ can be written in the form

$$
\begin{aligned}
& \frac{\partial Q_{13}}{\partial t}-L \Delta Q_{13}=F(\mathbf{Q}) Q_{13}+B Q_{12} Q_{23}, \\
& \frac{\partial Q_{23}}{\partial t}-L \Delta Q_{13}=G(\mathbf{Q}) Q_{23}+B Q_{12} Q_{13},
\end{aligned}
$$

where $F$ and $G$ are bounded functions by virtue of the $L^{\infty}$-bound above. We integrate by parts, use the fact that $Q_{13}=Q_{23}=0$ on $r=1$, and apply Gronwall's inequality to obtain

$$
\left(\int_{\Omega} Q_{13}^{2}+Q_{23}^{2} \mathrm{~d} V\right) \leq\left.\exp \left[\delta_{4} t\right]\left(\int_{\Omega} Q_{13}^{2}+Q_{23}^{2} \mathrm{~d} V\right)\right|_{t=0}=0,
$$

so that $Q_{13}=Q_{23}=0$ for all $t \geq 0$. 
4.1.1. Radially symmetric static solutions. We consider a particular class of planar critical points of the LdG energy on a disc, introduced in [15] for low temperatures (described by $A<0$ in (2.2)), referred to as radially symmetric solutions. The theoretical results in this section are a generalization of the results in [15] to the nematic-isotropic transition temperature. We work in a different temperature regime where the LdG bulk potential has two equal energy minima, and hence we cannot a priori assume that the results in $[15,26]$ apply to our case. These solutions are labelled by two order parameters $u$ and $v$ that depend only on the radial distance from the origin. We can perform some explicit analysis for these critical points, which are good examples of planar Q-tensors. We first show that there exist radially symmetric planar $(u, v)$-critical points of the LdG energy on a disc at the nematic-isotropic transition temperature, which are unstable in the sense that the second variation of the LdG energy is negative for admissible perturbations. We use these critical points to construct planar initial conditions for the LdG gradient flow model. We then use numerical simulations to corroborate our heuristics that the corresponding dynamic solutions quickly develop a radially symmetric nematic-isotropic interface (even when the initial data is not radially symmetric) and converge to $\mathbf{Q}_{1}$ everywhere away from the origin. Next we investigate the effect of small "nonplanar" perturbations of the planar initial conditions and show that the solution follows the planar dynamics for small but noticeable times, followed by an abrupt escape into the third dimension at $r=0$ and long-time convergence to $\mathbf{Q}_{2}$. As in [15], we study the LdG Euler-Lagrange equations,

$$
L \Delta \mathbf{Q}=\frac{B^{2}}{27 C} \mathbf{Q}-B\left(\mathbf{Q} \mathbf{Q}-\frac{|\mathbf{Q}|^{2}}{3} \mathbf{I}\right)+C|\mathbf{Q}|^{2} \mathbf{Q},
$$

and look for solutions of the form

$$
\mathbf{Q}=\frac{u(r)}{2}\left(\mathbf{n}_{1} \otimes \mathbf{n}_{1}-\mathbf{m} \otimes \mathbf{m}\right)+v(r)\left(\mathbf{p} \otimes \mathbf{p}-\frac{\mathbf{I}}{3}\right),
$$

where $\mathbf{m}=(-\sin \theta, \cos \theta, 0)$ and $\mathbf{p}=(0,0,1)$. The critical points $(4.7)$ are referred to as $k$-radially symmetric solutions in $[15,26]$ with $k=2$; we note that the authors normalize the tensors in (4.7), while we choose not to normalize the tensors for ease of presentation. It is straightforward to verify that solutions of the form (4.7) exist if the functions $u$ and $v$ satisfy the following system of coupled second-order ordinary differential equations:

$$
\begin{aligned}
& u^{\prime \prime}(r)+\frac{u^{\prime}(r)}{r}-\frac{4 u(r)}{r^{2}}=\frac{u}{L}\left(\frac{B^{2}}{27 C}+\frac{2}{3} B v+C\left(\frac{u^{2}}{2}+\frac{2 v^{2}}{3}\right)\right), \\
& v^{\prime \prime}(r)+\frac{v^{\prime}(r)}{r}=\frac{v}{L}\left(\frac{B^{2}}{27 C}-\frac{B v}{3}+C\left(\frac{u^{2}}{2}+\frac{2 v^{2}}{3}\right)\right)+\frac{1}{4 L} B u^{2},
\end{aligned}
$$

with $u(0)=v^{\prime}(0)=0$ with $u(R)=B / 3 C$ and $v(R)=-B / 6 C$, consistent with the boundary condition $\mathbf{Q}=\mathbf{Q}_{c}$ on $r=1$. As in [15], we can prove the existence of a solution pair $(u, v)$ of $(4.8)-(4.9)$ by appealing to a variational problem. Define the energy

$$
\begin{aligned}
\mathcal{E}(u, v)=\int_{0}^{1}( & \left(\frac{1}{4}\left(u^{\prime}\right)^{2}+\frac{1}{3}\left(v^{\prime}\right)^{2}+\frac{1}{r^{2}} u^{2}\right)+\frac{B^{2}}{54 C L}\left(\frac{u^{2}}{2}+\frac{2 v^{2}}{3}\right) \\
& \left.+\frac{C}{L}\left(\frac{u^{4}}{16}+\frac{u^{2} v^{2}}{6}+\frac{v^{4}}{9}\right)-\frac{B}{3 L} v\left(\frac{2 v^{2}}{9}-\frac{u^{2}}{2}\right)\right) r \mathrm{~d} r .
\end{aligned}
$$


This is the LdG energy of the $(u, v)$-ansatz in (4.7), defined on the admissible set $S=\left\{(u, v):[0,1] \rightarrow \mathbb{R}^{2} \mid \sqrt{r} u^{\prime}, \sqrt{r} v^{\prime}, u / \sqrt{r}, \sqrt{r} v \in L^{2}(0,1), u(1)=B / 3 C, v(1)=\right.$ $-B / 6 C\}$. The proof of the following lemma is standard and omitted here for brevity (see $[15])$.

Lemma 4.2. For each $L>0$ there exists a global minimizer $(u, v) \in\left[C^{\infty}(0,1) \cap\right.$ $C([0,1])] \times\left[C^{\infty}(0,1) \cap C^{1}([0,1])\right]$ of the energy (4.10) on $S$, which satisfies the ODEs for $u$ and $v$ in $(4.8)-(4.9)$.

Next, we look at some qualitative properties of the $(u, v)$-solutions. Similar questions have been addressed in the recent paper [26], in the temperature regime $A<0$, with the exception of the monotonicity argument in Lemma 4.4 below, and we reproduce all necessary details for completeness.

Lemma 4.3. Let $(u, v)$ be a global minimizer of the energy $\mathcal{E}$ in (4.10) subject to $u(1)=\frac{B}{3 C}$ and $v(1)=-\frac{B}{6 C}$. Then $0 \leq u \leq \frac{B}{3 C}$ and $-\frac{B}{6 C} \leq v \leq 0$ for $0 \leq r \leq 1$.

Proof. We can prove the nonnegativity of $u$ by following the arguments in $[15,26]$. (The authors use the symmetry $E[u, v]=E[-u, v]$ and the strong maximum principle for (4.8) to deduce that $u \geq 0$ since $u(1)>0$.) We assume $v\left(r_{1}\right)=v\left(r_{2}\right)=0$ and $v>0$ for $r_{1}<r<r_{2}$. Define the perturbation

$$
\bar{v}(r)= \begin{cases}v(r), & 0 \leq r \leq r_{1} \\ 0, & r_{1}<r<r_{2} \\ v(r), & r_{2} \leq r \leq 1\end{cases}
$$

A direct computation shows that

$$
E[u, v]-E[u, \bar{v}]=\int_{r_{1}}^{r_{2}} r \frac{v_{r}^{2}}{3}+\frac{r}{L} \frac{C v^{2}}{9}\left(v-\frac{B}{3 C}\right)^{2}+r \frac{u^{2}}{6 L}\left(B v+C v^{2}\right) \mathrm{d} r>0
$$

contradicting the global minimality of the pair $(u, v)$. Next, let us assume for a contradiction that the minimum value of $v:[0,1] \rightarrow \mathbb{R}$, denoted by $v_{\min }$, is attained at $r=r_{0}$, and $v_{\min }$ is less than $-\frac{B}{6 C}$; i.e., $v_{\min }<-\frac{B}{6 C}$ at $r=r_{0}$. At $r=r_{0}$, the left-hand side of (4.9) is nonnegative by definition of a minimum. From the maximum principle (see $[17,4]$ ), we have

$$
|\mathbf{Q}|^{2}=\frac{u^{2}}{2}+\frac{2 v^{2}}{3} \leq \frac{2}{3}\left(\frac{B^{2}}{9 C^{2}}\right)
$$

If $v_{\min }<-\frac{B}{6 C}$, then $u^{2}\left(r_{0}\right)<\frac{B^{2}}{9 C^{2}}$. Then for $v_{\min }<-\frac{B}{6 C}$ we have

$$
\begin{aligned}
& \frac{B^{2}}{27 C} v_{\text {min }}-\frac{B}{3} v_{\text {min }}^{2}+\frac{2 C}{3} v_{\text {min }}^{3}<-\frac{B^{3}}{54 C^{2}} \text { and } \\
& \frac{u^{2}}{4}\left(B+2 C v_{\text {min }}\right)<\frac{B^{3}}{54 C^{2}},
\end{aligned}
$$

so that the right-hand side of (4.9) is negative, yielding a contradiction. The result for $u$ follows in the same manner, using the bound for $v$ proven above.

We next show that $(u, v)$ are monotone functions, borrowing an idea from [27]. We make the elementary observation that $u^{\prime}>0$ for $0<r<\sigma$, since $u$ attains its minimum value at $r=0$,

$$
u_{\epsilon}(r)=u(r)+\epsilon \alpha(r), \quad v_{\epsilon}(r)=v(r)+\epsilon \beta(r),
$$


with $\alpha(1)=\beta(1)=0$. In this case the second variation is given by

$$
\begin{aligned}
\delta^{2} E[\alpha, \beta]:= & \int_{0}^{1} r\left[\frac{\alpha_{r}^{2}}{4}+\frac{\beta_{r}^{2}}{3}+\frac{\alpha^{2}}{r^{2}}\right]+\frac{r}{L}\left[\frac{B^{2}}{27 C} \frac{\beta^{2}}{3}-\frac{2 B v \beta^{2}}{9}+\frac{2 C}{3} v^{2} \beta^{2}\right] \mathrm{d} r \\
& +\int_{0}^{1} \frac{r}{L}\left[\frac{C}{6} u^{2} \beta^{2}+\frac{B}{6} v \alpha^{2}+\frac{C}{6} v^{2} \alpha^{2}+\frac{B u \alpha \beta}{3}+\frac{2 C u v \alpha \beta}{3}\right] \mathrm{d} r \\
& +\int_{0}^{1} \frac{r}{L}\left[\frac{B^{2}}{108 C} \alpha^{2}+\frac{3 C}{8} u^{2} \alpha^{2}\right] \mathrm{d} r .
\end{aligned}
$$

In particular, $\delta^{2} E[\alpha, \beta] \geq 0$ for all admissible $\alpha, \beta$ by the global minimality of $(u, v)$.

Lemma 4.4. Let $(u, v)$ be a global minimizer of the energy in (4.10). Then $u^{\prime}>0$ for $r>0$ and $v^{\prime}<0$ for $r>0$.

Proof. We assume for a contradiction that $u$ and $v$ are not monotone, so that there exist points $r_{1}, r_{2}, r_{3}, r_{4}$ with $r_{1}, r_{2} \in(0,1), r_{3} \in[0,1)$ such that

$$
\begin{aligned}
& u^{\prime}\left(r_{1}\right)=u^{\prime}\left(r_{2}\right)=0, \quad u^{\prime}<0 \text { for } r_{1}<r<r_{2}, \\
& v^{\prime}\left(r_{3}\right)=v^{\prime}\left(r_{4}\right)=0, \quad v^{\prime}>0 \text { for } r_{3}<r<r_{4} .
\end{aligned}
$$

We differentiate the ODEs for $u$ and $v$ in (4.8)-(4.9), multiply by $r u^{\prime}$ and $r v^{\prime}$ respectively, and integrate over $r \in\left[r_{1}, r_{2}\right]$ and $r \in\left[r_{3}, r_{4}\right]$ to get the following equalities:

$$
\begin{aligned}
\int_{r_{1}}^{r_{2}} \frac{r}{4}\left(u^{\prime \prime}\right)^{2}+\frac{5}{4 r}\left(u^{\prime}\right)^{2}-\frac{2}{r^{2}} u u^{\prime}+ & \frac{r}{L} \\
\quad & {\left[\frac{B^{2}}{108 C}\left(u^{\prime}\right)^{2}+\frac{3 C}{8} u^{2}\left(u^{\prime}\right)^{2}\right.} \\
& \left.+\frac{C}{6}\left(u^{\prime}\right)^{2} v^{2}+\frac{B}{6} v\left(u^{\prime}\right)^{2}+\frac{u u^{\prime} v^{\prime}}{6}(B+2 C v)\right] \mathrm{d} r=0
\end{aligned}
$$

and

$$
\begin{gathered}
\int_{r_{3}}^{r_{4}} \frac{r}{3}\left(v^{\prime \prime}\right)^{2}+ \\
+\frac{\left(v^{\prime}\right)^{2}}{3 r}+\frac{r}{L}\left[\frac{B^{2}}{81 C}\left(v^{\prime}\right)^{2}-\frac{2 B}{9} v\left(v^{\prime}\right)^{2}+\frac{2 C}{3} v^{2}\left(v^{\prime}\right)^{2}\right] \\
+\frac{r}{L}\left[\frac{C}{6} u^{2}\left(v^{\prime}\right)^{2}+\frac{u u^{\prime} v^{\prime}}{6}(B+2 C v)\right] \mathrm{d} r=0 .
\end{gathered}
$$

We define the perturbations $\alpha, \beta$ as follows:

$$
\alpha(r)=\left\{\begin{array}{ll}
0 & \text { if } u^{\prime} \geq 0, \\
u^{\prime} & \text { if } u^{\prime}<0,
\end{array} \quad \beta(r)= \begin{cases}0 & \text { if } v^{\prime} \leq 0 \\
v^{\prime} & \text { if } v^{\prime}>0\end{cases}\right.
$$

These perturbations satisfy $\alpha(1)=\beta(1)=0$, since $u$ and $v$ attain their maximum and minimum values on $r=1$, respectively. Substituting $(\alpha, \beta)$ into (4.16) and using (4.18)-(4.19), we obtain $\delta^{2} E[\alpha, \beta]<0$ and the required contradiction.

Finally, we demonstrate that this class of critical points is unstable in the static sense, at the nematic-isotropic transition temperature. We consider a perturbation about the critical point in (4.7), $\mathbf{W}_{i j}=\mathbf{Q}_{i j}+\epsilon \mathbf{V}_{i j}$ with $\mathbf{V}_{i j}=0$ on $r=1$, and compute the second variation of the LdG energy about this critical point as shown below:

$$
\delta^{2} I=\iiint \frac{1}{2}|\nabla \mathbf{V}|^{2}+\frac{A}{2 L}|\mathbf{V}|^{2}-\frac{B}{L} \mathbf{Q}_{i j} \mathbf{V}_{j p} \mathbf{V}_{p i}+\frac{C}{L}(\mathbf{Q} \cdot \mathbf{V})^{2}+\frac{C}{2 L}|\mathbf{Q}|^{2}|\mathbf{V}|^{2} \mathrm{~d} V .
$$


We simply use the perturbation

$$
\mathbf{V}=\frac{100 r^{2}\left(1-r^{2}\right)^{2}}{\left(1+100 r^{2}\right)}\left(\mathbf{n}_{1} \otimes \mathbf{p}+\mathbf{p} \otimes \mathbf{n}_{1}\right)
$$

in (4.21). This integral is evaluated numerically using numerical solutions to (4.8)(4.9) and $\delta^{2} I<0$ for $\log _{10} L<-1.6$, as illustrated in the graph of Figure 4.1.

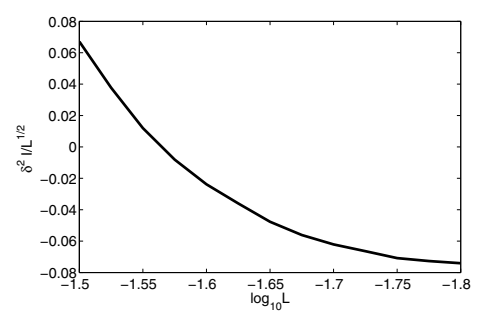

FIG. 4.1. $\delta^{2} I$ for various $L$.

4.2. Numerical simulations. We numerically solve the LdG gradient flow system in (3.24)-(3.28) on a disc subject to $\mathbf{Q}=\mathbf{Q}_{c}$ on $r=1$. We take $R$ to be the disc radius in the definition of the dimensionless variables, and the parameter values $(R$, $L, B, C)$ are as in section 3 . We take $\tilde{L}=0.01$ in this section, unless stated otherwise. The focus is on the distinction between planar and nonplanar initial conditions. Based on the heuristics presented in section 4.1.1, we argue that all dynamic solutions develop an interface separating an isotropic core, centered at $r=0$, from an ordered uniaxial nematic phase away from $r=0$. For planar initial conditions the interface persists for all times and the dynamic solution has an isotropic core at $r=0$ for all times, whereas for nonplanar initial conditions the interface collapses at $r=0$ and the dynamic solution relaxes into the uniaxial state $\mathbf{Q}_{2}$ in (4.3) for long times.

First, we consider planar $(u, v)$-type initial conditions of the form

$$
\mathbf{Q}(\mathbf{r}, 0)=\frac{u(r, 0)}{2}\left(\mathbf{n}_{1} \otimes \mathbf{n}_{1}-\mathbf{m} \otimes \mathbf{m}\right)+v(r, 0)\left(\mathbf{p} \otimes \mathbf{p}-\frac{\mathbf{I}}{3}\right),
$$

where $\mathbf{m}=(-\sin \theta, \cos \theta, 0), \mathbf{p}=(0,0,1)$, and $\mathbf{I}=\mathbf{n}_{1} \otimes \mathbf{n}_{1}+\mathbf{m} \otimes \mathbf{m}+\mathbf{p} \otimes \mathbf{p}$. Recall that our boundary conditions enforce $u=B / 3 C$ and $v=-B / 6 C$ on $r=1$. We let $u(r, 0)$ and $v(r, 0)$ have the interface structure

$$
u(r, 0)=\frac{B}{6 C}\left(1+\tanh \left(\frac{r-u_{0}}{\sqrt{\tilde{L}}}\right)\right), \quad v(r, 0)=-\frac{B}{12 C}\left(1+\tanh \left(\frac{r-v_{0}}{\sqrt{\tilde{L}}}\right)\right)
$$

for various values of $u_{0}$ and $v_{0}$. It is worth noting that the corresponding dynamic solution is given by

$$
\mathbf{Q}(\mathbf{r}, t)=\frac{u(r, t)}{2}\left(\mathbf{n}_{1} \otimes \mathbf{n}_{1}-\mathbf{m} \otimes \mathbf{m}\right)+v(r, t)\left(\mathbf{p} \otimes \mathbf{p}-\frac{\mathbf{I}}{3}\right)
$$

if the dynamic order parameters satisfy

$$
\begin{aligned}
& \gamma u_{t}=u^{\prime \prime}(r)+\frac{u^{\prime}(r)}{r}-\frac{4 u(r)}{r^{2}}-\frac{u}{\tilde{L}}\left(\frac{B^{2}}{27 C}+\frac{2}{3} B v+C\left(\frac{u^{2}}{2}+\frac{2 v^{2}}{3}\right)\right), \\
& \gamma v_{t}=v^{\prime \prime}(r)+\frac{v^{\prime}(r)}{r}-\frac{v}{\tilde{L}}\left(\frac{B^{2}}{27 C}-\frac{B v}{3}+C\left(\frac{u^{2}}{2}+\frac{2 v^{2}}{3}\right)\right)-\frac{1}{4 \tilde{L}} B u^{2} .
\end{aligned}
$$




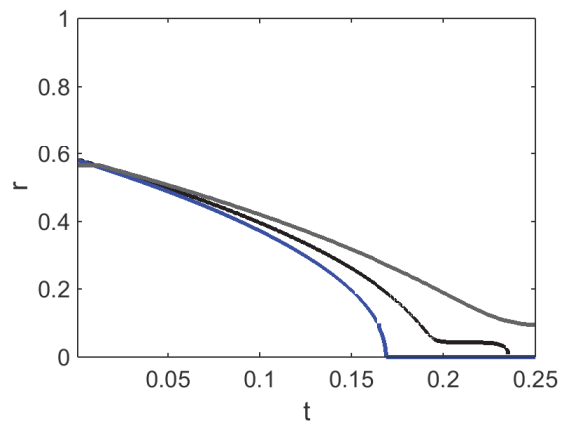

FIG. 4.2. Interface position of dynamic solution (4.24) with initial condition (4.23), with $u_{0}=$ $0.6, v_{0}=0.4$ for $\tilde{L}=0.05$ (grey) and $\tilde{L}=0.01$ (black) compared to motion by mean curvature (blue).
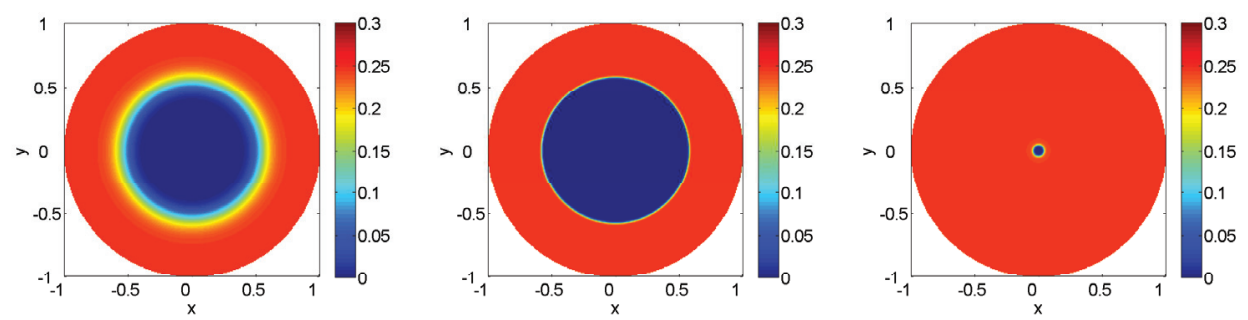

FIG. 4.3. $|\boldsymbol{Q}(\mathbf{r}, t)|^{2}$ for $u_{0}=0.6, v_{0}=0.4$ for the initial condition (4.23), at $t=0, t=10^{-5}$, and $t=0.25$. The spatial resolution is $h=\frac{1}{256}$.

From Proposition 2.1, this is the unique solution for this model problem. In fact, we can go further and exploit the methods in [28] to compare the nematic-isotropic interface motion in (4.24)-(4.26) with mean curvature motion. As in section 3, we cannot quote results from [28] since the dynamic equations (4.24)-(4.26) differ from the GL model in [28] by the additional term $-4 u / r^{2}$ in (4.25) above. However, for $\tilde{L}$ sufficiently small this term may be controllable, and in Figure 4.2 we plot the numerically computed interface location (plot $r^{*}(t)$ such that $|\mathbf{Q}(\mathbf{r}, t)|^{2}<\frac{1}{3} h_{+}^{2}$ for $\left.r<r^{*}(t)\right)$ and find good agreement with mean curvature propagation for small times.

A typical solution with an initial condition of the form (4.23) is shown in Figure 4.3. If $u_{0} \neq v_{0}$, then $\mathbf{Q}(\mathbf{r}, 0)$ is necessarily biaxial, but it is hard to see the biaxial character of the initial data by looking at $|\boldsymbol{Q}|^{2}$. In order to see the rapid relaxation to uniaxiality, we plot the eigenvalues of the dynamic solution, $\mathbf{Q}(\mathbf{r}, t)$, as a function of time (see Figure 4.4). Varying the values of $u_{0}$ and $v_{0}$ does not change the qualitative dynamics: $\mathbf{Q}(\mathbf{r}, t)$ quickly becomes uniaxial for all choices of $u_{0}$ and $v_{0}$, within numerical resolution. The dynamic solution develops a radially symmetric interface separating the isotropic core, centered at $r=0$, from an ordered uniaxial nematic state (away from $r=0$ ), and the interface equilibrates near $r=0$ for long times. We have numerically computed the tensor-difference, $\mathbf{Q}(\mathbf{r}, t)-\mathbf{Q}_{1}$, as a function of time (where $\mathbf{Q}_{1}$ is introduced in (4.3)) and find that $\mathbf{Q}(\mathbf{r}, t) \rightarrow \mathbf{Q}_{1}$ everywhere away from $r=0$, as expected. One component of this tensor difference is plotted in Figure 4.5. 



FIG. 4.4. Eigenvalues of $\boldsymbol{Q}(\mathbf{r}, t)$ for initial condition (4.23) with $u_{0}=0.6, v_{0}=0.4$, at $t=0$, $t=10^{-5}$, and $t=0.25$.
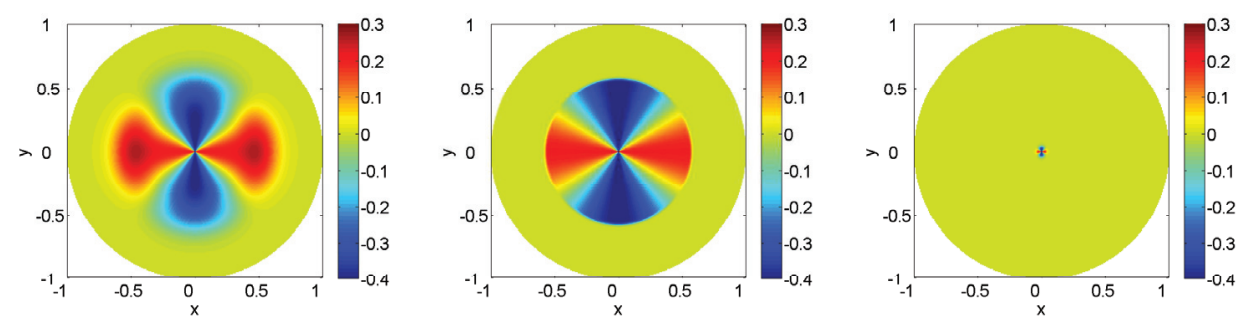

FIG. 4.5. $Q_{11}(\mathbf{r}, t)-\frac{B}{3 C}\left(\frac{x^{2}}{r^{2}}-\frac{1}{3}\right)$ on the cross section of the cylinder at $z=0$ for the initial condition (4.23) with $u_{0}=0.6, v_{0}=0.4$, at $t=0, t=10^{-5}$, and $t=0.25$.
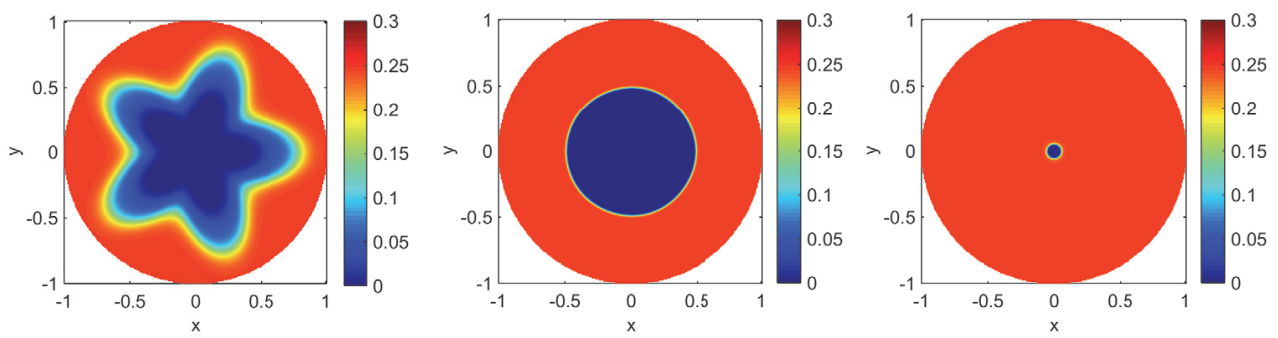

FIG. 4.6. $|\boldsymbol{Q}(\mathbf{r}, t)|^{2}$ on $z=0$ for initial condition (4.27) at $t=0, t=0.06$, and $t=0.2$. The spatial resolution is $h=\frac{1}{256}$.

Next, we consider an initial condition of the form (4.23) with

$$
\begin{aligned}
& u(r, \theta, 0)=\frac{B}{6 C}\left(1+\tanh \left(\frac{r-0.6(1+0.25 \sin 5 \theta)}{\sqrt{\tilde{L}}}\right)\right), \\
& v(r, \theta, 0)=-\frac{B}{12 C}\left(1+\tanh \left(\frac{r-0.4(1+0.25 \sin 5 \theta)}{\sqrt{\tilde{L}}}\right)\right) .
\end{aligned}
$$

This is again a planar initial condition with an interesting star-shaped nematicisotropic interface that relaxes into a radially symmetric nematic-isotropic interface. The subsequent dynamics is then indistinguishable from the case discussed above; this is illustrated by Figures 4.6 and 4.7 .

Finally, we consider a small perturbation to the planar $(u, v)$-initial conditions, 

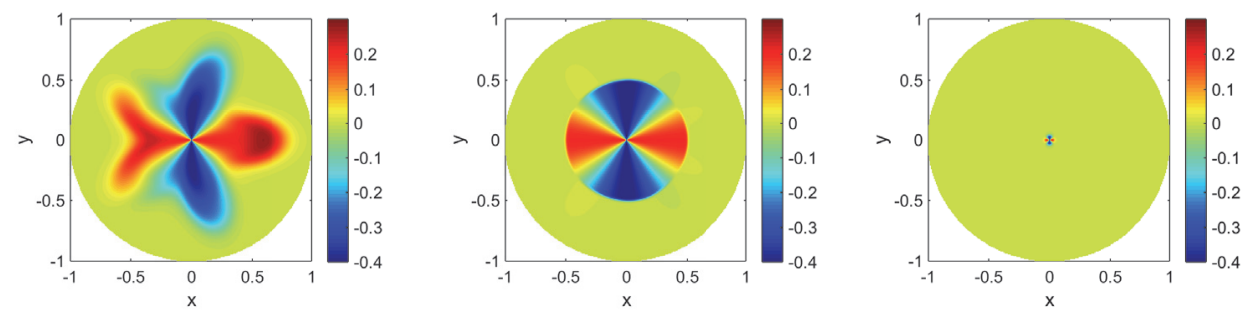

FIG. 4.7. $Q_{11}(\mathbf{r}, t)-\frac{B}{3 C}\left(\frac{x^{2}}{r^{2}}-\frac{1}{3}\right)$ on the unit disc for the initial condition (4.27) at $t=0$, $t=0.06$, and $t=0.2$.
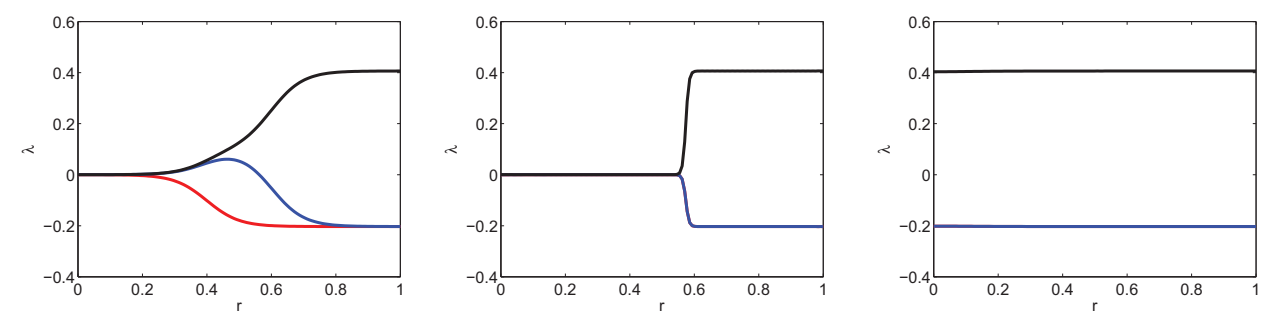

FIG. 4.8. Spatial dependence of eigenvalues of $\boldsymbol{Q}(\mathbf{r}, t)$ at $t=0, t=0.001$, and $t=0.25$ for initial condition (4.28) with $u_{0}=0.6, v_{0}=0.4$.
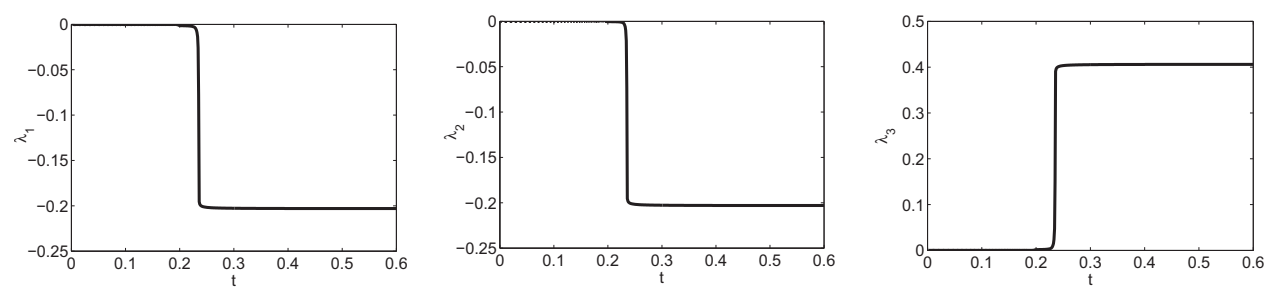

FIG. 4.9. Time evolution of eigenvalues of $\boldsymbol{Q}(\mathbf{r}, t)$ at the origin for initial condition (4.28) with $u_{0}=0.6, v_{0}=0.4$.

which yields nonplanar initial conditions. Let

$$
\mathbf{Q}(r, 0)=u(r, 0)\left(\mathbf{n} \otimes \mathbf{n}-\frac{\boldsymbol{I}_{2}}{2}\right)+v(r, 0)\left(\mathbf{p} \otimes \mathbf{p}-\frac{\boldsymbol{I}}{3}\right),
$$

where

$$
\begin{aligned}
& \boldsymbol{n}=\left(\sqrt{\left(1-\epsilon^{2}(1-r)^{2}\right)} \cos \theta, \sqrt{\left(1-\epsilon^{2}(1-r)^{2}\right)} \sin \theta, \epsilon(1-r)\right), \\
& \mathbf{I}_{2}=\mathbf{n}_{1} \otimes \mathbf{n}_{1}+\mathbf{m} \otimes \mathbf{m},
\end{aligned}
$$

and $\boldsymbol{p}$ is as before. The functions $u(r, 0)$ and $v(r, 0)$ are as defined previously. As before, the dynamic solution quickly becomes uniaxial (within numerical resolution) irrespective of $u_{0}$ and $v_{0}$ and develops a well-defined interface separating an interior region, with $\mathbf{Q}=0$ near $r=0$, from an ordered uniaxial nematic state elsewhere. This interface propagates inward, but instead of being arrested at a small distance from the origin, the interface collapses at the origin and the dynamic solution relaxes to $\mathbf{Q}_{2}$ in (4.3). In particular, $|\mathbf{Q}(\mathbf{r}, t)|^{2} \rightarrow \frac{2}{3} h_{+}^{2}$ uniformly for large times. Figures 4.8 and 4.9 show the time snapshots of the spatial distribution of eigenvalues, and 

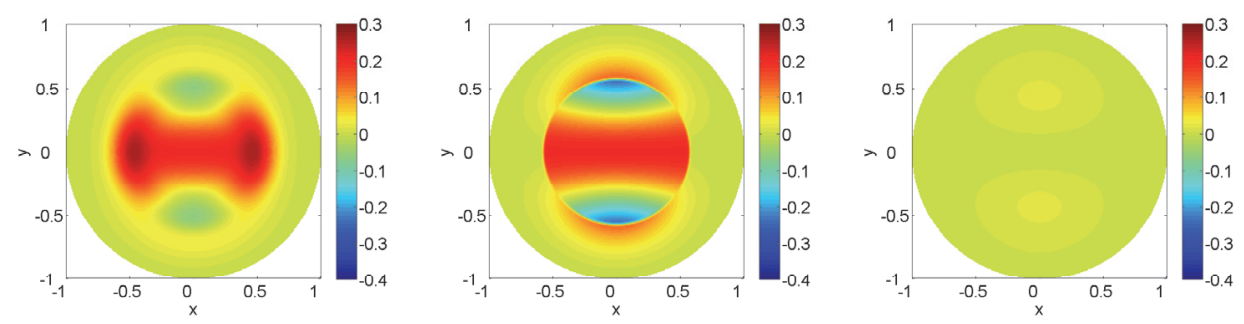

FIG. 4.10. $Q_{11}(\mathbf{r}, t)-\frac{B}{3 C}\left(4 x^{2} /\left(1+r^{2}\right)^{2}-\frac{1}{3}\right)$ for initial condition $(4.28)$ with $u_{0}=0.6, v_{0}=0.4$ at $t=0, t=0.001$, and $t=0.6$.

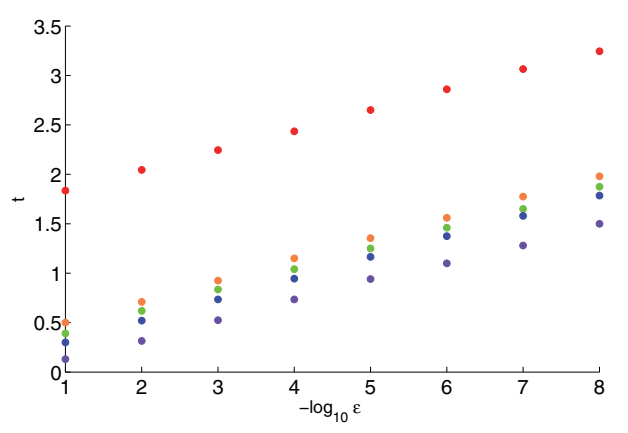

FIG. 4.11. Time $\left(t^{*}\right)$ at which the interface is lost for various $\epsilon$ and $u_{0}$ and $v_{0} ; u_{0}=0.1$ (purple), $u_{0}=0.4$ (blue), $u_{0}=0.5$ (green), $u_{0}=0.6$ (orange), and $u_{0}=0.9$ (red); $v_{0}=1-u_{0}$ for initial condition (4.28) and $\tilde{L}=0.05$.

the time evolution of the eigenvalues at $r=0$, showing the convergence to a uniform uniaxial solution. Figure 4.10 shows snapshots of the relaxation of one component of $\mathbf{Q}$ to the corresponding $\mathbf{Q}_{2}$-component.

We also study how the initial nonplanarity (as measured by $\epsilon$ ) affects the characteristic relaxation time to $\mathbf{Q}_{2}$. We observe that the modulus, $|\mathbf{Q}|^{2}(0, t)$, jumps abruptly from zero to $\frac{2}{3} h_{+}^{2}$ at some critical time. Let $t^{*}$ be the first time for which

$$
\left|\mathbf{Q}\left(0, t^{*}\right)\right|^{2}>\frac{1}{3} h_{+}^{2},
$$

and we associate $t^{*}$ with the loss of interface structure. Figure 4.11 plots $t^{*}$ as a function of $-\log _{10} \epsilon$ for various $u_{0}$ and $v_{0}$, and we find that $t^{*} \propto-\log _{10} \epsilon$. This can give quantitative estimates for the real-time persistence of nematic-isotropic interfaces and their experimental relevance for model problems with nonplanar initial conditions.

5. Biaxial boundary conditions on a disc. The Dirichlet conditions in sections 3 and 4 are uniaxial minima of the bulk potential, $f_{B}$, and are referred to as minimal boundary conditions. In this section, we employ a biaxial planar boundary condition that is not a minimum of $f_{B}$ at the nematic-isotropic transition temperature, given by

$$
\mathbf{Q}_{b}=\frac{B}{3 C}\left(\mathbf{n}_{1} \otimes \mathbf{n}_{1}-\mathbf{m} \otimes \mathbf{m}\right) .
$$

This boundary condition is maximally biaxial with a zero eigenvalue, i.e., $\operatorname{tr} \mathbf{Q}_{b}^{3}=0$. We refer to $\mathbf{Q}_{b}$ as a nonminimal Dirichlet condition. From [29], we expect that 
LdG energy minimizers, subject to a boundary condition of this form, converge in an appropriate sense to a limiting harmonic map that is a minimum of the bulk potential almost everywhere and develop a boundary layer near $r=1$ to match $\mathbf{Q}_{b}$, in the vanishing elastic constant limit.

We study 2D and 3D dynamic solutions separately. A 2D solution is a symmetric and traceless $2 \times 2$ matrix [18], and in such cases, we study maps from the disc to a $2 \mathrm{D}$ target space with just two degrees of freedom. A 3D solution is a symmetric, traceless $3 \times 3$ matrix, and in such cases, we study maps from a $2 \mathrm{D}$ domain into a $5 \mathrm{D}$ target space.

We start this section with a discussion of the $2 \mathrm{D}$ case; $2 \mathrm{D} \mathbf{Q}$-matrices have $\operatorname{tr} \mathbf{Q}^{3}=$ 0 and the corresponding evolution law simplifies to

$$
\mathbf{Q}_{t}=L \Delta \mathbf{Q}-A \mathbf{Q}-C|\mathbf{Q}|^{2} \mathbf{Q} .
$$

The simplest 2D dynamic solution, consistent with (5.1), is

$$
\mathbf{Q}(\mathbf{r}, t)=s(r, t)\left(\mathbf{n}_{1} \otimes \mathbf{n}_{1}-\mathbf{m} \otimes \mathbf{m}\right)
$$

with $r^{2}=x^{2}+y^{2}$. It is simple to check that the gradient flow model (5.2) admits a solution of the form (5.3) if the function $s(r, t)$ is a solution of

$$
\gamma s_{t}=\left\{s_{r r}+\frac{s_{r}}{r}-\frac{4 s}{r^{2}}\right\}-\frac{s}{L}\left(\frac{B^{2}}{27 C}+2 C s^{2}\right)
$$

with fixed boundary conditions

$$
s(0, t)=0, \quad s(1, t)=\frac{B}{3 C},
$$

for all $t \geq 0$. The evolution equation (5.4) is simply the gradient flow model for the functional

$$
I[s]:=\int_{0}^{1}\left[r\left(\frac{d s}{d r}\right)^{2}+\frac{2 s^{2}}{r}\right]+\frac{r}{L}\left(\frac{B^{2}}{27 C} s^{2}+\frac{C}{4} s^{4}\right) \mathrm{d} r,
$$

and given a smooth solution, $s(r, t)$ of $(5.4)-(5.5)$ with suitable initial conditions, (5.3) is the unique 2D solution. Further, the 2D potential has an isolated minimum at $s=0$ (see (5.6)), and hence we expect that any dynamic solution of (5.2) has an outward-propagating interface that separates an almost isotropic core around $r=0$ from the Dirichlet boundary condition at $r=1$. The interface equilibrates near $r=1$, followed by a sharp boundary layer to match the fixed boundary condition.

Next, we present some heuristics for 3D dynamic solutions with a planar initial condition of the form

$$
\mathbf{Q}(\mathbf{r}, 0)=s(r, 0)\left(\mathbf{n}_{1} \otimes \mathbf{n}_{1}-\mathbf{m} \otimes \mathbf{m}\right),
$$

where $s(r, 0)$ has an interface structure, i.e.,

$$
s(r, 0)= \begin{cases}0, & r<r_{0}, \\ \frac{B}{3 C}, & r_{0}<r \leq 1,\end{cases}
$$

for some $r_{0} \in(0,1)$. Based on the analysis in section 4.1.1, all dynamic solutions remain planar with $Q_{13}=Q_{23}=0$. Hence, we expect $\mathbf{Q}(\mathbf{r}, t)$ (for long times) to have 
an isotropic core centered at $r=0$, and $\mathbf{Q}(\mathbf{r}, t)$ converges to $\mathbf{Q}_{1}$ (introduced in (4.3)) away from $r=0$, with a boundary layer near $r=1$ to match the Dirichlet condition. However, we speculate that there is a second scenario for nonminimal boundary conditions as in (5.1), which is not observed for minimal boundary conditions as in section 4 . If $1-r_{0}$ is sufficiently small, 3D solutions may exhibit an outward-growing isotropic core since the isotropic phase is also a minimizer of the bulk potential, and this scenario may be energetically favorable. All dynamic solutions with nonminimal boundary conditions develop a boundary layer near $r=1$, which has an energetic cost. However, solutions with minimal boundary conditions (as in sections 3 and 4) do not have boundary layers near $r=1$, and in such cases it is energetically preferable either to have a localized core of reduced order near $r=0$ (as for planar 3D solutions) or to have uniform order throughout the disc (as for nonplanar 3D solutions).

Thus, we expect sharp contrast in the behavior of $2 \mathrm{D}$ and $3 \mathrm{D}$ solutions. The 2D dynamic solutions have little nematic order, being largely isotropic or close to isotropic, except near $r=1$, and 3D dynamic solutions are largely uniaxial with perfect nematic ordering (for at least a range of values of $r_{0}$ ), except near $r=0$ and $r=1$. It is not clear whether $2 \mathrm{D}$ dynamic solutions could be physically relevant for certain materials or temperature regimes. In the next section, we present numerical results to validate our heuristics above.

5.1. Numerical simulations with biaxial boundary conditions. We numerically solve (3.24)-(3.28) on a disc with the Dirichlet condition (5.1) on $r=1$. All other parameter values are as in section 4.2 , with $\tilde{L}=0.01$. The planar initial condition is as in (5.7) with

$$
s(r)=\frac{h_{+}}{2}\left(1+\tanh \left(\frac{r-r_{0}}{\sqrt{\tilde{L}}}\right)\right) .
$$

In the case of $r_{0}=0.5$, the solution quickly becomes almost uniaxial in the interior by developing an inwards-propagating well-defined nematic-isotropic interface. This is illustrated by a plot of eigenvalue evolution in Figure 5.1. The solution converges to $\mathbf{Q}_{1}(\mathbf{r}, t)$ (see (4.3)) away from $r=0$ for long times, with the core of reduced order near $r=0$ and a thin boundary layer near $r=1$, as displayed in Figure 5.2. This is as expected from the numerical results presented in section 4 .
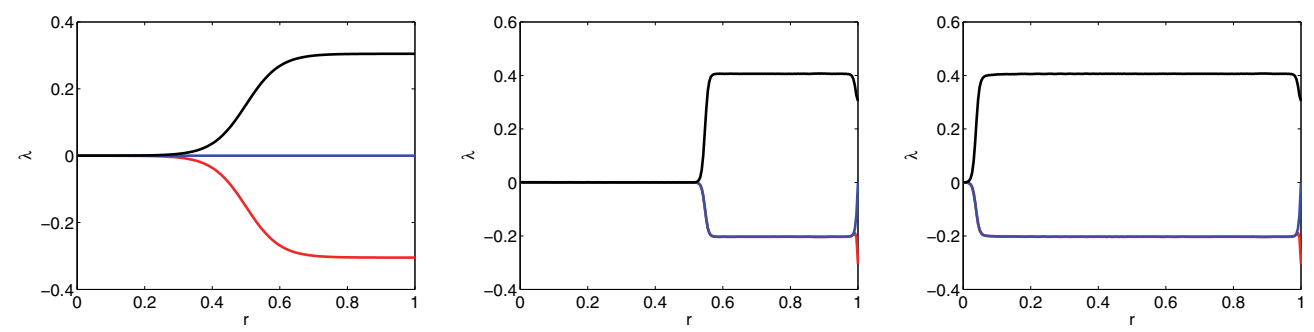

FIG. 5.1. Eigenvalues of $\boldsymbol{Q}(\mathbf{r}, t)$ for initial condition (5.7) (with $r_{0}=0.5$ ) at $t=0, t=0.001$, and $t=0.25$.

Next, we consider $r_{0}=0.925$ and observe a different behavior; the interface evolves so that there is a thin boundary layer near $r=1$ with a large, almost isotropic, core in the interior. This is best illustrated with radial profiles of $|\boldsymbol{Q}|^{2}$, as seen in Figure 5.3. 

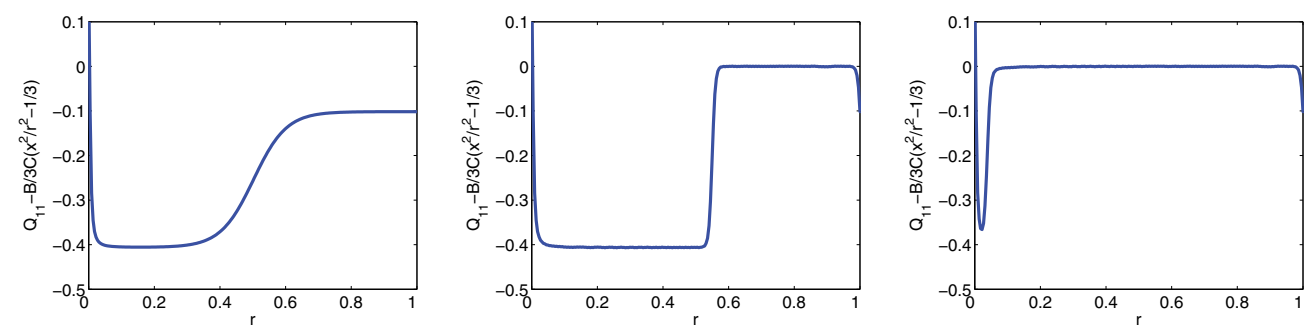

FIG. 5.2. Radial profile of $Q_{11}(\mathbf{r}, t)-\frac{B}{3 C}\left(\frac{x^{2}}{r^{2}}-\frac{1}{3}\right)$ for $\theta=0$ for initial condition (5.7) (with $\left.r_{0}=0.5\right)$ at $t=0, t=0.001$, and $t=0.25$.
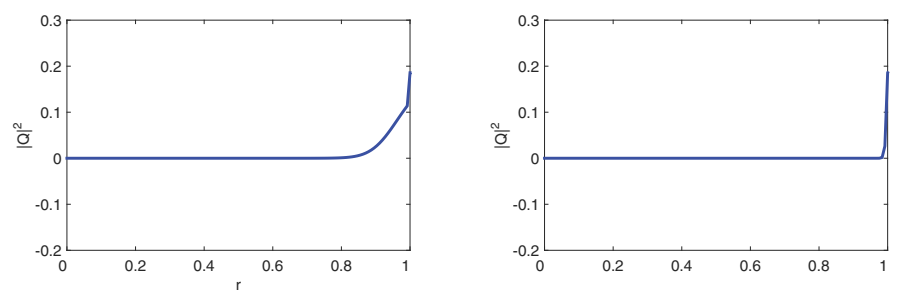

FIG. 5.3. Radial profile of $|\boldsymbol{Q}(\mathbf{r}, t)|^{2}$ for initial condition (5.7) with $r_{0}=0.925$, for $t=0$ and $t=0.25$.
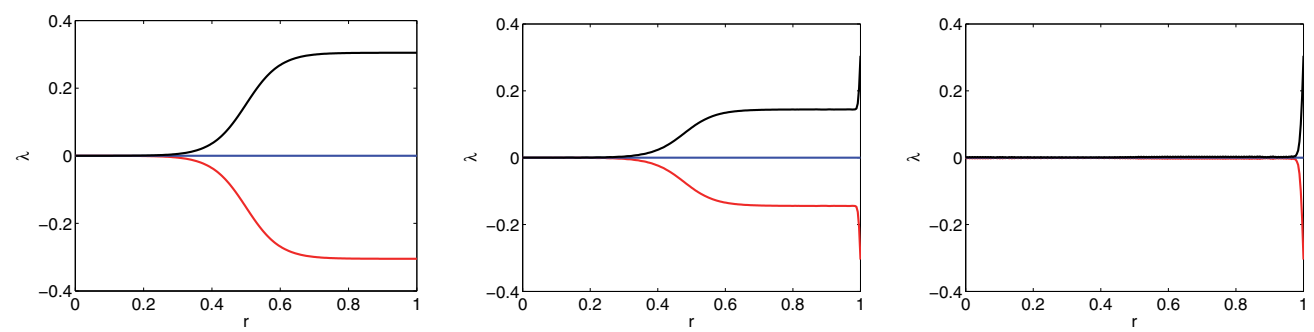

FIG. 5.4. Eigenvalues of $\mathbf{Q}(\mathbf{r}, t)$ in the $2 D$ model, for initial condition (5.7) at $t=0, t=$ $2 \times 10^{-5}$, and $t=2 \times 10^{-4}$.

We compare the 3D solutions above with 2D solutions for the same system (5.2). We work with planar initial conditions (5.7), with $s(r)$ as before. The initial interface grows rapidly to yield an almost entirely isotropic interior with a thin boundary layer near $r=1$. This is illustrated by the eigenvalue evolution in Figure 5.4, thus corroborating our heuristics and analytical reasoning in the previous section.

6. Conclusions. We focus on the gradient flow model for the LdG energy on prototype geometries, such as a droplet and a disc, with Dirichlet boundary conditions and various initial conditions at the nematic-isotropic transition temperature. In section 3 , we consider the model problem of a 3D droplet of radius $R$, with radial boundary conditions. In the case of uniaxial radially symmetric initial conditions with a nematic-isotropic front structure, we adapt Ginzburg-Landau methods from $[10,11]$ to prove that the nematic-isotropic interface propagates according to mean curvature in the $\frac{L}{R^{2}} \frac{C}{B^{2}} \rightarrow 0$ limit, for small times. However, the qualitative dynamics seem universal for a large class of radially symmetric and nonsymmetric uniaxial and biaxial initial conditions, and the long-time dynamics converges to the classical 
RH solution, which has been numerically demonstrated to be a global LdG energy minimizer in this regime.

In sections 4 and 5, we focus on dynamic solutions on a disc. Our results are largely numerical and complemented by heuristics and analytical reasoning. We demonstrate how a choice of planar or nonplanar initial condition can influence the long-time dynamic behavior. Planar initial conditions generate planar dynamic solutions with an isotropic core around the center for all times, whereas nonplanar solutions follow the planar dynamics for a length of time before relaxing into an uniaxial state of perfect order for long times. In section 5, we look at nonminimal boundary conditions. Nonminimal boundary conditions allow for dynamic scenarios outside the scope of minimal boundary conditions, and since minimal boundary conditions are an idealization, nonminimal Dirichlet conditions can be physically relevant too.

The long-time dynamics can be understood in terms of local and global minimizers, or in some cases critical points, of the LdG energy. In cases where the LdG critical points exhibit an nematic-isotropic interface, this interface may be localized with little effect on global properties. Our numerical results show that a large class of physically relevant LC model problems can exhibit a well-defined nematic-isotropic interface for a length of time (see Figures 3.2 and 4.2), and these results give insight into how boundary and initial conditions can be used to yield either largely disordered or ordered nematic profiles. A natural next step is to rigorously analyze front formation and propagation with generic nonminimal boundary conditions and with more general LdG energy functionals, including those with a sixth-order bulk potential that allow for biaxial minima. We will report on these developments in the future.

Acknowledgments. We thank Heiko Gimperlein, Giacomo Canevari, and the anonymous referees for helpful comments.

\section{REFERENCES}

[1] P.-G. De Gennes And J. Prost, The Physics of Liquid Crystals, vol. 23, Clarendon Press, Oxford, UK, 1993.

[2] E.G. VIRGA, Variational Theories for Liquid Crystals, vol. 8, CRC Press, Boca Raton, FL, 1995.

[3] A. Majumdar, The Landau-de Gennes theory of nematic liquid crystals: Uniaxiality versus biaxiality, Comm. Pure \& Appl. Anal., 11 (2012), pp. 1303-1337.

[4] A. Majumdar and A. Zarnescu, Landau-de Gennes theory of nematic liquid crystals: The Oseen-Frank limit and beyond, Arch. Ration. Mech. Anal., 196 (2010), pp. 227-280.

[5] M.A. Peletier, Energies, Gradient Flows, and Large Deviations: A Modelling, lecture notes, 2011.

[6] V. Popa-Nita and T.J. Sluckin, Kinetics of the nematic-isotropic interface, J. Phys. II, 6 (1996), pp. 873-884.

[7] V. Popa-Nita, T.J. Sluckin, And A.A. Wheeler, Statics and kinetics at the nematic-isotropic interface: Effects of biaxiality, J. Phys. II, 7 (1997), pp. 1225-1243.

[8] M. Fei, W. Wang, P. Zhang, and Z. Zhang, Dynamics of the nematic-isotropic sharp interface for the liquid crystal, SIAM J. Appl. Math., 75 (2015), pp. 1700-1724.

[9] A. Sengupta, U. Tkalec, M. Ravnik, J.M. Yeomans, C. Bahr, and S. Herminghaus, Liquid crystal microfluidics for tunable flow shaping, Phys. Rev. Lett., 110 (2013), 048303.

[10] L. Bronsard and R.V. Kohn, Motion by mean curvature as the singular limit of GinzburgLandau dynamics, J. Differential Equations, 90 (1991), pp. 211-237.

[11] L. Bronsard and B. Stoth, On the existence of high multiplicity interfaces, Math. Res. Lett., 3 (1996), pp. 41-50.

[12] A. Majumdar, The radial-hedgehog solution in Landau-de Gennes' theory for nematic liquid crystals, European J. Appl. Math., 23 (2012), pp. 61-97.

[13] S. Mkaddem and E.C. Gartland, JR., Fine structure of defects in radial nematic droplets, Phys. Rev. E, 62 (2000), pp. 6694-6705. 
[14] D. Henao And A. Majumdar, Symmetry of uniaxial global Landau-de Gennes minimizers in the theory of nematic liquid crystals, SIAM J. Math. Anal., 44 (2012), pp. 3217-3241.

[15] G. Di Fratta, J.M. Robbins, V. Slastikov, and A. Zarnescu, Half-integer point defects in the q-tensor theory of nematic liquid crystals, J. Nonlinear Sci., 26 (2016), pp. 121-140.

[16] N.J. Mottram and C.J.P. Newton, Introduction to Q-Tensor Theory, preprint, arXiv:1409.3542, 2014.

[17] A. MAJumdar, Equilibrium order parameters of nematic liquid crystals in the Landau-de Gennes theory, European J. Appl. Math., 21 (2010), pp. 181-203.

[18] A. Majumdar, J. Ockendon, P. Howell, and E. SurovyatKina, Transitions through critical temperatures in nematic liquid crystals, Phys. Rev. E, 88 (2013), 022501.

[19] J. Morgan, Global existence for semilinear parabolic systems, SIAM J. Math. Anal., 20 (1989), pp. $1128-1144$.

[20] A. Majumdar, Order parameters in the Landau-de Gennes theory-The static and dynamic scenarios, Liquid Crystals, 38 (2011), pp. 169-181.

[21] P. Sternberg, The effect of a singular perturbation on nonconvex variational problems, Arch. Ration. Mech. Anal., 101 (1988), pp. 209-260.

[22] X. Zhong, A new high-order immersed interface method for solving elliptic equations with imbedded interface of discontinuity, J. Comput. Phys., 225 (2007), pp. 1066-1099.

[23] D. Golovaty and J.A. Montero, On minimizers of a Landau-de Gennes energy functional on planar domains, Arch. Ration. Mech. Anal., 213 (2014), pp. 447-490.

[24] F. Bethuel, H. Brezis, B.D. Coleman, And F. HÉlein, Bifurcation analysis of minimizing harmonic maps describing the equilibrium of nematic phases between cylinders, Arch. Ration. Mech. Anal., 118 (1992), pp. 149-168.

[25] M. Renardy and R.C. Rogers, An Introduction to Partial Differential Equations, Springer Science \& Business Media 13, Springer, New York, 2004.

[26] R. Ignat, L. Nguyen, V. Slastikov, and A. Zarnescu, Instability of point defects in a twodimensional nematic liquid crystal model, in Ann. Inst. H. Poincaré Anal. Non Linéare (C), Elsevier, Paris, 2015.

[27] X. LAmY, Some properties of the nematic radial hedgehog in the Landau-de Gennes theory, J. Math. Anal. Appl., 397 (2013), pp. 586-594.

[28] L. Bronsard and B. Stoth, The singular limit of a vector-valued reaction-diffusion process, Trans. Amer. Math. Soc., 350 (1998), pp. 4931-4953.

[29] I. ShAFriR, On a class of singular perturbation problems, in Stationary Partial Differential Equations, 1, 2004, pp. 297-383. 